\title{
Deutschlands Rolle im internationalen Handel mit konventionellen Waffen und Rüstungsgütern: Sind wir die „Waffenkammer der Welt"?
}

https://doi.org/10.1515/sirius-2018-2004

Kurzfassung: Der Artikel setzt sich mit der Rolle der Bundesrepublik im internationalen Handel mit konventionellen Waffen auseinander. Dabei werden drei immer wieder $\mathrm{zu}$ vernehmende Behauptungen anhand der vorhandenen statistischen Daten und wissenschaftlichen Analysen überprüft: (1) Deutschland sei drittgrößter Exporteur von Waffen und Rüstungsgütern; (2) Deutschland sei weltweit der zweitgrößte Exporteur von Kleinwaffen; und (3) deutsche Rüstungsexporte würden zur Entstehung, zur Eskalation und Verlängerung von Kriegen sowie zu Rüstungswettläufen beitragen. Der Beitrag gelangt zu dem Ergebnis, dass keine dieser Behauptungen einer kritischen Prüfung standhält. Bedauerlich ist nur, wie sehr falsche Behauptungen und Unterstellungen selbst von seriösen Medien übernommen werden und in den politischen Diskurs eingehen.

Schlüsselwörter: Rüstungsexporte, Bundesrepublik Deutschland, internationaler Waffenhandel, Rüstungsindustrie, Militärhilfe

Abstract: The article deals with the relative importance of Germany in the international trade with conventional weapons and armaments. It addresses three claims that are dominating the political debate: (1) Germany was the third largest arms exporter on a global scale; (2) Germany is supposed to be the second largest exporter of small arms in the world; and (3) Germany bore responsibility for the emergence, the escalation and the prolongation of international and civil wars as well as for arms races in many regions. The article arrives at the conclusion that none of these claims does stand up to an empirical examination. Notwithstanding the facts, these claims are still dominating the political discourse in Germany.

Keywords: arms exports, Germany, international trade in conventional weapons, military aid

*Kontakt: Prof. Dr. Joachim Krause, Direktor des Instituts für Sicherheitspolitik an der Universität Kiel und Herausgeber von SIRIUS,

E-Mail: JKrause@politik.uni-kiel.de

\section{Einleitung}

Es gibt in keinem Land der Welt eine derart (selbst-)kritische politische Debatte zu Rüstungsexporten und deren mutmaßlichen Konsequenzen wie in Deutschland. Immer dann, wenn die Bundesregierung aktuelle Zahlen zu den erteilten Genehmigungen und den tatsächlich erfolgten Exporten von Waffen und sonstigen Rüstungsgütern vorlegt, wird in Medien und Politik entweder mit Erleichterung ein Rückgang oder mit Empörung eine Zunahme registriert. Nehmen Exportgenehmigungen zu, dann werden Forderungen nach mehr Transparenz, nach neuen Gesetzen oder nach einer grundsätzlich neuen Rüstungsexportpolitik vorgelegt. Die Deutschen - so scheint es leiden an ihren Rüstungsexporten wie kein anderes Volk.

Unter „Rüstungsexporten“ werden kommerzielle und nicht-kommerzielle (meist im Rahmen von staatlich gewährter Militärhilfe) Exporte von Kriegswaffen sowie von relevanten Komponenten von Kriegswaffen (unter anderem auch Antriebsaggregate, Radar- und Feuerleitsysteme) und von Anlagen zur Herstellung von Kriegswaffen bezeichnet (,sonstige Rüstungsgüter“). Teilweise werden auch Exporte von Handfeuerwaffen mitgezählt, die nicht oder nicht primär für militärische Zwecke Verwendung finden. Oft wird als Sammelbegriff für alle Arten von wehrtechnischen Exporten auch der Terminus „Rüstungstransfers" benutzt. Von einem Transfer spricht man dann, wenn etwas tatsächlich von einem Land in ein anderes überführt wurde.

Die Kritik an Rüstungsexporten beziehungsweise an der politischen Genehmigungspraxis der Bundesregierung hat eine lange Tradition und wird seit den 1970erJahren durch Analysen aus der Friedensforschung mehr oder weniger seriös untermauert. In den 1960er-Jahren war die kritische Verfolgung der deutschen Rüstungsexport- und Militärhilfepraxis geboten, denn auf diesem sensiblen Feld wurden von der damaligen Bundesregierung schwerwiegende Fehler begangen. Dazu gehörten nicht nur unbedachte Waffenlieferungen in Spannungsgebiete, sondern insbesondere die weite Verbreitung des Sturm- 
gewehrs G3 der Firma Heckler \& Koch. ${ }^{1}$ Seit den 1980erJahren hingegen haben sich eigentlich alle Bundesregierungen (gleich welcher politischen Richtung) darum bemüht, eine vorsichtige und zurückhaltende Rüstungsexportpolitik $\mathrm{zu}$ betreiben. ${ }^{2}$ Auffallend ist jedoch, wie wenig die hiesige politische Debatte zu Rüstungsexporten diese Wandlungen berücksichtigt. Vielmehr beherrschen Behauptungen und Feststellungen die Debatte, die den Regierungsanspruch widerlegen sollen. In der Regel wird dabei auf die angeblich führende Rolle Deutschlands im internationalen Waffenhandel verwiesen und es wird behauptet, dass deutsche Waffen maßgeblich in Kriegsgebieten zum Einsatz kämen. ${ }^{3}$ Die entsprechenden Feststellungen stammen zumeist aus der kritischen Friedensforschung, oftmals aber auch von einer rüstungskritischen Gegenöffentlichkeit, die teilweise tief im linksextremen Milieu verortet ist. Deutlich erkennbar ist die Dominanz des rüstungskritischen Narrativs, verfolgt man die Debatte in beiden christlichen Kirchen zu Rüstungsexporten. ${ }^{4}$ Selbst professionelle Medien, wie die öffentlichrechtlichen Sender ARD und ZDF und die Redaktionen von SPIEGEL, ZEIT, Handelsblatt, Süddeutscher Zeitung, Welt oder Frankfurter Allgemeiner Zeitung, die gewohnt sind, Daten und Informationen genau zu prüfen, übernehmen bei Rüstungsexporten völlig unkritisch Daten und Bewertungen aus diesem Umfeld.

Doch wie groß ist tatsächlich der Realitätsgehalt dieser Behauptungen und Feststellungen? Im Folgenden soll dieser Frage anhand einer kritischen Sichtung der einschlägigen Datenlage und der wissenschaftlichen Literatur nachgegangen werden. Dabei geht es im Einzelnen um den Wahrheitsgehalt von drei Behauptungen: (1) Deutschland sei der drittgrößte Waffenexporteur der Welt (hinter den USA und Russland und noch vor Großbritannien und Frankreich); (2) Deutschland sei der weltweit zweitgrößte Exporteur von Kleinwaffen (hinter den USA); und (3) Exporte deutscher Waffen und Rüstungsgüter würden zur Entstehung, zur Eskalation und zur Verlängerung von Konflikten und zu Rüstungswettläufen beitragen. Wie glaubhaft sind die oben angeführten Behauptungen? Spiegeln sie die Realität wider? Oder sind sie Übertreibungen?

1 Vgl. Haftendorn 1971, Haftendorn 1972, Albrecht 1972.

2 Vgl. Krause 2013.

3 Vgl. Aken/Lurz 2012, Grässlin 2013, Friedrichs 2012.

4 Seit vielen Jahren betreiben beide Kirchen eine gemeinsame Kommission, die jährlich Berichte zur Rüstungsexportpolitik veröffentlichen und in der Regel die deutsche Politik schwer kritisieren, vgl. zuletzt GKKE 2016. Aber auch die Kirchentage lassen erkennen, dass sie von Überlegungen dominiert werden, die von den linken Rüstungskritikern stammen.

\section{Die wichtigsten wissenschaftlichen Quellen}

Mit internationalen Waffenexporten befasst sich eine Reihe von wissenschaftlichen Instituten und staatlichen beziehungsweise nichtstaatlichen Einrichtungen, die als wissenschaftsnah $\mathrm{zu}$ charakterisieren sind, weil sie mit Mitteln der wissenschaftlichen Analyse arbeiten. ${ }^{5}$ Vor allem die folgenden Einrichtungen sind hier zu erwähnen, weil sie eigenständige Recherchen anstellen und diese publizieren:

- Das internationale Friedensforschungsinstitut Stockholm International Peace Research Institute (SIPRI), das seit seiner Gründung 1966 öffentlich zugängliche Daten zu Rüstungstransfers (meist Großtransfers) sammelt und auswertet. Früher wurden die Ergebnisse nur im Rahmen des SIPRI-Jahrbuchs veröffentlicht, heute bietet SIPRI auch im Internet eine umfängliche und benutzerfreundliche Datenbank an. ${ }^{6}$ Die Analysen und Statistiken von SIPRI basieren auf öffentlich zugänglichen Angaben über Großwaffengeschäfte. Das bedeutet, es werden Informationen aus wehrtechnischen Zeitschriften und Informationsdiensten ausgewertet, ebenso Pressemeldungen, öffentliche Stellungnahmen und Geschäftsberichte großer Konzerne. Diese Meldungen werden gesammelt, aufbereitet und systematisiert. Die Liste der Geschäfte findet man auf einer öffentlich zugänglichen Datenbank von SIPRI. Unter Großwaffenexporte werden nicht nur Exporte von Kriegsschiffen, Militärflugzeugen, Raketen, gepanzerten Fahrzeugen, Artilleriesystemen und Luftabwehrsystemen gerechnet, sondern auch Systeme zur U-Boot-Bekämpfung, Komponenten von gepanzerten Fahrzeugen und Kriegsschiffen sowie Antriebsaggregate für Kriegsschiffe und militärische Flugzeuge, Radargeräte und Flugleitsysteme sowie militärisch genutzte Satelliten. Alle registrierten Transfers werden durch ein Expertengremium begutachtet und mit einem mutmaßlichen Preis versehen. Auf dieser Basis wird für jedes Land eine Ausfuhr- und Einfuhrstatistik erstellt. Früher wurden die Preise in laufenden und inflationsbereinigten US-Dollar erstellt, mittlerweile wird ein sogenannter Trendindikatorenwert (trend-indicator value, TIV) angegeben, der nur noch Trends angibt, aber keine in Dollar oder Euro übersetzbare Zahlen.

5 Für einen Überblick zu den unterschiedlichen Methoden und Verfahren vgl. Garcia-Alonso/Levine 2007.

6 Vgl. https://www.sipri.org/databases/armstransfers, ansonsten zuletzt SIPRI 2017. 
- SIPRI ist nicht das einzige Institut, das öffentlich verfügbare Daten über Transfers von Großwaffensystemen sammelt und aufbereitet. Ähnlich gehen das in London beheimatete Internationale Institut für Strategische Studien (International Institute for Strategic Studies, IISS) vor sowie der private Informationsdienst Jane's.

- Der Wissenschaftliche Dienst des US-amerikanischen Kongresses (Congressional Research Service, CRS) veröffentlicht regelmäßig Überblicksanalysen zum internationalen Waffentransfer, insbesondere in Drittweltstaaten. Der CRS führt dazu keine eigenen Recherchen durch, sondern übernimmt Analysen eines ungenannt bleibend wollenden US-Geheimdienstes (vermutlich CIA). Dieser Nachrichtendienst macht zwar keine Angaben zur Methode, geht aber offenbar in ähnlicher Weise vor wie SIPRI. Die dort erfassten Transfers sind allerdings umfangreicher und involvieren auch Zulieferungen von Komponenten sowie die Gewährung von Militärhilfe. Die Daten werden in laufenden und konstanten US-Dollars wiedergegeben. Die Angaben sind grobe Schätzwerte, sie werden auf volle Hundert Millionen Dollar entweder auf- oder abgerundet. ${ }^{7}$ Früher wurden diese Daten durch die inzwischen aufgelöste US Arms Control and Disarmament Agency (ACDA) beziehungsweise durch das Department of State veröffentlicht.

- Der Rechnungshof des US-amerikanischen Kongresses (Government Accountability Office, GAO) legt ebenfalls wiederholt Analysen vor, die sich mit Aspekten des internationalen Waffentransfers (auch von Kleinwaffen) befassen und auf Angaben basieren, die USRegierungsbehörden zur Verfügung gestellt haben.

- Der Small Arms Survey ist ein von dem kanadischen Politikwissenschaftler Keith Krause begründetes Forschungsprojekt am Graduate Institute for International and Development Studies in Genf. Das Forschungsprojekt befasst sich mit allen Aspekten, die kleine und leichte Waffen betreffen. Dazu zählen Analysen, die die Lage in bestimmten Regionen oder Kriegsschauplätzen behandeln, ebenso aber auch Untersuchungen $\mathrm{zu}$ technologischen Entwicklungen sowie $\mathrm{zu}$ Transfers von kleinen und leichten Waffen. Die Projektmitarbeiter kooperieren mit Nichtregierungsorganisationen, mit internationalen Organisationen und Regierungen. Ihre Publikationen finden sich auf ihrer Website, darunter auch der jährlich erscheinende Small Arms Survey. ${ }^{8}$

7 Zuletzt siehe Theohary 2015 und Theohary 2016.

8 Vgl. http://www.smallarmssurvey.org.
- Bei dem Projekt Conflict Armaments Research (CAR) handelt es sich um eine in London basierte Forschungsgruppe, die sich mit der Analyse von Kleinwaffen, Munition und anderen militärischen Einsatzmitteln befasst und vornehmlich deren Transfers und Auftauchen bei Konflikten, Kriegen und Bürgerkriegen im Blick hat. Die Forschungsgruppe existiert seit 2011 und wird überwiegend aus Mitteln der EU und der Schweizer Bundesregierung finanziert. Die Forschungsgruppe arbeitet teilweise wie eine NichtRegierungsorganisation und versucht, durch Recherchen vor Ort Einblicke in die Art und Weise zu gewinnen, wie Kriegsparteien und Terrororganisationen an Waffen gelangen. Untersuchungsteams waren bereits in über 20 Ländern auf der Suche nach Waffenlagern und Produktionsstätten für Waffen und Munition. Die Ergebnisse der Analysen werden auf der Website der Gruppe veröffentlicht. ${ }^{9}$ Dort finden sich ausführliche Hinweise zu den Methoden und zur Vorgehensweise von CAR.

- Eine wichtige Quelle sind auch die Berichte von Expertengruppen, die im Rahmen der Überwachung von Waffenembargos des Sicherheitsrates eingesetzt werden und - sofern sie die entsprechenden Möglichkeiten haben - Waffenarsenale oder Waffenfunde inspizieren beziehungsweise Fotos, Zeugenaussagen und Überreste von Kämpfen untersuchen. ${ }^{10}$

- Es liegen weitere Berichte internationaler NGOs oder Forschungsgruppen vor, die vornehmlich auf die Einhaltung von Menschenrechten, die Berichterstattung über Konflikte sowie die Beobachtung illegaler Wirtschaftstätigkeiten fokussiert sind, dabei aber auch Informationen über Teilaspekte des internationalen Waffenhandels vermitteln. Dazu gehört unter anderem die in Washington, D.C. sitzende Global Financial Integrity, die sich mit illegalen Geschäften und auch Waffentransfers befasst. ${ }^{11}$

In Deutschland gibt es kein Forschungsinstitut, das dauerhaft eigenständige Recherchen über Rüstungstransfers anstellt. Lediglich das Bonn International Center for Conversion (BICC) führt Untersuchungen durch, die sich mit der allgemeinen politischen und wirtschaftlichen Lage der Empfängerstaaten deutscher Rüstungsexporte befassen. Die Ergebnisse werden auf einer Website wiedergegeben, ${ }^{12}$ neben weitergehenden Informationen zu Rüstungstrans-

\footnotetext{
9 Vgl. http://www.conflictarm.com. 10 Vgl. LeBrun/Rigual 2016.

11 Vgl. http://www.gfintegrity.org.

12 Vgl. http://www.ruestungsexport.info.
} 
fers sowie $\mathrm{zu}$ vereinzelten Untersuchungen von Einrichtungen aus dem Bereich der Friedensforschung.

\section{Ist Deutschland drittgrößter Waffenexporteur der Welt?}

Deutschland gehört zu denjenigen Ländern, die mittlerweile sehr detailliert über Rüstungstransfers berichten. In dem jährlich erscheinenden Bericht der Bundesregierung zu Rüstungsexporten wird genau aufgeführt, wie viele Genehmigungen für den Export von Kriegswaffen und sonstigen Rüstungsgütern erteilt worden sind und in welcher Größenordnung dann auch tatsächlich Exporte von Kriegswaffen stattgefunden haben. Dabei wird zwischen Transfers in Länder der EU, der NATO und denen gleichgestellte auf der einen sowie Transfers in Drittstaaten auf der anderen Seite differenziert. In den vergangenen zehn Jahren lag das Niveau der Exporte deutscher Kriegswaffen zwischen einer und 2,5 Milliarden Euro pro Jahr (Tabelle 1). Lediglich im Jahr 2016 wurden Kriegswaffen im Wert von 2,5 Milliarden Euro exportiert. Kriegswaffenexporte entsprachen somit zwischen ein und zwei Promille der deutschen Exporte im gleichen Zeitraum. ${ }^{13} \mathrm{Im}$ Jahre 2016 wurden Genehmigungen über die Ausfuhr von Kriegswaffen und sonstigen Rüstungsgütern im Wert von 6,848 Milliarden Euro erteilt, was allerdings keine Aussage über die tatsächlich erfolgten Transfers zulässt. ${ }^{14}$

Tab. 1: Deutsche Kriegswaffenexporte 2006 bis 2016

\begin{tabular}{llll}
\hline Jahr & $\begin{array}{l}\text { Gesamtwert in } \\
\text { Mio. } €\end{array}$ & $\begin{array}{l}\text { Davon: kommer- } \\
\text { zielle Exporte } \\
\text { in Drittländer in } \\
\text { Mio } €\end{array}$ & $\begin{array}{l}\text { Anteil am } \\
\text { deutschen } \\
\text { Gesamtexport } \\
\text { in Prozent }\end{array}$ \\
\hline 2006 & $1.374,2$ & 423,5 & 0,15 \\
2007 & $1.510,1$ & 275,8 & 0,16 \\
2008 & $1.427,2$ & 388,8 & 0,14 \\
2009 & $1.338,8$ & 179,7 & 0,17 \\
2010 & $2.119,0$ & 453,0 & 0,22 \\
2011 & $1.284,7$ & 842,8 & 0,12 \\
2012 & 946,0 & 559,1 & 0,09 \\
2013 & 956,6 & 568,1 & 0,09 \\
2014 & $1.826,0$ & $1.338,0$ & 0,16 \\
2015 & $1.554,9$ & $1.173,0$ & 0,13 \\
2016 & $2.501,8$ & $2.297,0$ & 0,21 \\
\hline
\end{tabular}

Quelle: Bundesministerium für Wirtschaft und Energie, 2017, $38 \mathrm{f}$.
Die Angaben der Bundesregierung sagen noch nichts über die Vergleichbarkeit mit anderen Staaten aus. Die Behauptung, wonach Deutschland drittgrößter Waffenexporteur der Welt sei, wird in der Regel mit Statistiken von SIPRI belegt. Das war für viele Jahre tatsächlich der Fall, seit 2012 hat Deutschland in den SIPRI-Statistiken diese Position allerdings an Frankreich und China abgegeben und rangiert nunmehr auf Platz 5 (Tabelle 2 und Tabelle 3), laut neuesten Angaben ist es für 2017 auf Platz 4 gerückt. Aber seit den 1980er-Jahren war es - von wenigen Jahren abgesehen - geradezu eine Konstante, dass die Bundesrepublik Deutschland nach den USA und Russland als weltweit drittgrößter Waffenexporteur galt. Gefolgt wurde Deutschland meist mit knappem Abstand von Frankreich. Großbritannien lag den SIPRI-Daten zufolge weit dahinter, oft nur mit einem Exportvolumen halb so groß wie das deutsche.

Tab. 2: Deutschlands Bedeutung als Rüstungsexporteur nach SIPRI (2008-2013)

\begin{tabular}{ll}
\hline $\begin{array}{l}\text { Exporteur von Großwaffen- } \\
\text { systemen }\end{array}$ & Anteil am Welthandel in Prozent \\
\hline 1. USA & 30 \\
2. Russland & 26 \\
3. Deutschland & 7 \\
4. Frankreich & 6 \\
5. China & 5 \\
6. Großbritannien & 4 \\
7. Spanien & 3 \\
8. Italien & 2 \\
9. Ukraine & 2 \\
10. Israel & 2 \\
\hline
\end{tabular}

Tab. 3: Deutschlands Bedeutung als Rüstungsexporteur nach SIPRI (2011-2015)

\begin{tabular}{lc}
\hline $\begin{array}{l}\text { Exporteur von Großwaffen- } \\
\text { systemen }\end{array}$ & Anteil am Welthandel in Prozent \\
\hline 1. USA & 33,0 \\
2. Russland & 25,0 \\
3. China & 5,9 \\
4. Frankreich & 5,6 \\
5. Deutschland & 4,7 \\
6. Großbritannien & 4,5 \\
7. Spanien & 3,5 \\
8. Italien & 2,7 \\
9. Ukraine & 2,6 \\
10. Niederlande & 2,0 \\
\hline
\end{tabular}


Die beim CRS veröffentlichten Statistiken kommen zu ähnlichen Aussagen wie SIPRI, sind allerdings teilweise detaillierter. Auch bei diesen Angaben ist erkennbar, dass Deutschland nicht die Nummer drei im internationalen Waffenhandel ist, sondern knapp hinter Großbritannien auf Platz 5 liegt (siehe Tabelle 4).

Tab. 4: Deutschlands Bedeutung als Rüstungsexporteur nach Angaben der US-Regierung (2008-2015)

\begin{tabular}{lcc}
\hline Land & $\begin{array}{l}\text { Rüstungstransfers } \\
\text { in US-Dollar }\end{array}$ & $\begin{array}{l}\text { Davon an } \\
\text { Entwicklungsländer } \\
\text { in Prozent }\end{array}$ \\
\hline 1. USA & 117,275 & 66,5 \\
2. Russland & 66,5 & 93,0 \\
3. Frankreich & 29,3 & 62,0 \\
4. Großbritannien & 22,2 & 50,0 \\
5. Deutschland & 22,0 & 44,5 \\
6. China & 18,5 & 100,0 \\
7. Italien & 13,2 & 42,0 \\
\hline
\end{tabular}

Das IISS publiziert mittlerweile nur noch die Angaben über bekannt gewordene Rüstungsdeals (nicht nur grenzüberschreitende Transfers), wobei die Empfänger (Regierungen) und die Lieferanten (die beteiligten Firmen) genannt werden. ${ }^{15}$ Das IISS nimmt keine Bewertungen der Transfers im Sinne von pricetags vor und übernimmt auch nicht mehr externe Bewertungen (wie die des CRS oder von SIPRI) über die Entwicklungstendenzen des globalen Marktes für Rüstungsgüter. Anders agiert der private Informationsdienst Jane's, der auch derartige Statistiken führt, diese aber nicht veröffentlicht, sondern nur für teures Geld Privatkunden zur Verfügung stellt, ohne Quellen und Methoden kenntlich zu machen. Lediglich in Pressemeldungen wird gelegentlich über Ergebnisse berichtet, ohne dass erkennbar ist, wie die Analytiker von Jane's zu diesen Erkenntnissen gekommen sind. ${ }^{16}$

15 IISS 2017a, jedes der regionalen Kapitel der Military Balance enthält eine Aufstellung „selected arms procurements and deliveries“.

16 Im Juni 2016 wurde im Jahresbericht von Jane’s gemeldet, dass Deutschland erneut drittgrößter Waffenexporteur geworden sei - mit Zahlen, die in keiner Weise den Angaben der Bundesregierung entsprachen, und es war auch nicht erkennbar, wie der Branchendienst zu dieser Schlussfolgerung gekommen war. Auffällig war nur, wie die Meldung, wonach Deutschland der drittgrößte Waffenexporteur weltweit sei, von den deutschen Medien ungeprüft übernommen und teilweise mit reißerischem Unterton vorgestellt wurde. Vgl. „Deutschland ist drittgrößter Waffenexporteur der Welt“, Die Welt, 13. 6. 2016; „Waffenexporteure: Drei Nationen bewaffnen die Welt“, Wirtschaftswoche, 3. 7. 2016; „Deutschlands Waffenexporte: Ein Mordsgeschäft“, taz, 13. 6. 2016; „Rüstungsexporte: Der Tod kommt aus Deutschland“, Handelsblatt, 13. 6. 2016; „Deutschland ist weltweit drittgrößter
Die Zurückhaltung des IISS bei der Aufstellung von Statistiken über den Welthandel mit Großwaffensystemen beruht auf grundsätzlichen methodischen Überlegungen. Das IISS geht davon aus, dass es nicht vertretbar ist, Aussagen über die Marktplatzierung einzelner Staaten bei Großwaffentransfers und deren Komponenten gemessen in Währungseinheiten oder Trendindikatoren auf der Basis öffentlich verfügbarer Angaben vorzunehmen. Die Gründe für diese Skepsis sind tatsächlich schwerwiegend. Sie werden in der deutschen politischen Debatte so gut wie nicht reflektiert:

1. Die Herstellung von Waffensystemen und Rüstungsgütern ist ebenso wie die anderer komplexer technischer Systeme in einem Maße Gegenstand grenzübergreifender Arbeitsteilung geworden, dass die Zuordnung von Rüstungstransfers zu einem Herstellerland immer weniger möglich wird. Wehrtechnische Produktion findet unter Bedingungen der Globalisierung zunehmend in transnationalen Zusammenhängen statt. ${ }^{17}$ Dies liegt an der multinationalen Struktur vieler Unternehmen, die aus dem Zusammenschluss von Firmen unterschiedlicher Nationalität entstanden sind (wie Airbus), die sich zur Auslagerung von Produktion in Empfängerländer oder in Länder mit geringeren Produktionskosten entschlossen haben (wie BAE Systems) oder die sich im Rahmen von Offset-Vereinbarungen auf komplexe Arbeitsteilungen mit Empfängerländern und dort befindlichen Firmen einlassen mussten. Eine Folge der Transnationalisierung der wehrtechnischen Produktion ist, dass viele Rüstungsgüter (meistens Waffenkomponenten, Einzelsysteme, Teile von Antriebsaggregaten etc.) mehrfach exportiert und re-importiert werden, sodass aus der Exportstatistik nicht mehr ermessen werden kann, wie groß der tatsächliche Transfer militärischer Machtressourcen im Einzelfall gewesen ist. Auch werden die Netze der Zulieferfirmen immer internationalisierter und umfassen zunehmend Güter und Technologien, die für militärische wie zivile Zwecke hergestellt werden. Dadurch wird es schwer, eine Abgrenzung zwischen militärischen und nicht-militärischen Transfers vorzunehmen. Eine andere Folge ist, dass viele Exporte von Großwaffensystemen gar nicht mehr aufgeführt werden, weil die Güter teilweise oder gar überwie-

Waffenexporteur“, ZEIT Online, 13. Juni 2016 http://www.zeit.de/ wirtschaft/2016-06/ruestungsindustrie-waffenexporte-deutschlanddrittgroesster-waffenexporteur.

17 Zur Transnationalisierung der Rüstungsindustrie vgl. Bitzinger 1994; Guay 1998; Defense Science Board 1999; Hayward 2000; Neal/ Taylor 2001; James 2002; Dowdall 2004; Guay 2007; Garcia 2009; Hayward 2009; Udis 2009; Bitzinger 2009; Hanel 2012; Devore 2013. 
gend im Empfängerland hergestellt werden. Ein Beispiel ist der britische multinationale Rüstungskonzern BAE Systems, der mit einem wehrtechnischen Umsatz von über 21 Milliarden Euro per annum und 83.000 Mitarbeitern weltweit der drittgrößte Spieler ist und mehr Waffensysteme produziert und exportiert als die gesamte deutsche wehrtechnische Industrie zusammen (siehe Tabelle 5). BAE Systems liefert Großwaffensysteme an viele Länder, oft in transnationaler oder ausgelagerter Produktion. Bei den Statistiken von SIPRI (weniger bei den US-amerikanischen Regierungsdaten) liegt Großbritannien dennoch als Rüstungsexporteur weit hinter Deutschland.

2. In der Methode von SIPRI sowie der der US-amerikanischen Regierung (und vermutlich auch der von Jane's) sind erhebliche Ansätze zur Fehlbewertung enthalten. Das betrifft zum einen die Informationsgewinnung. Durch die Beschränkung auf öffentlich zugängliche Informationen ist es unvermeidlich, dass diejenigen Firmen, die in Ländern operieren, die ein hohes Maß an Transparenz vorschreiben, stärker ins Licht geraten als Firmen, die in nicht-demokratischen und autoritären Staaten liegen. Von daher ist es kein Wunder, wenn in diesen Statistiken westliche Demokratien und Rechtsstaaten als die größten Rüstungsexporteure auftauchen. Zum anderen sind die Bewertungsverfahren für den Preis von Großwaffentransfers nicht transparent und beruhen offensichtlich auf einer Einschätzung technischer Parameter durch ungenannt bleibende Experten. Diese Schätzungen berücksichtigen weder konkrete transnationale Wertschöpfungsketten noch verhindern sie, dass Vorurteile zum Tragen kommen.

3. Die Angaben von SIPRI und vom CRS zu Großwaffentransfers reflektieren offenkundig nur einen Teil (vermutlich nicht mal die Hälfte) des internationalen Handels mit Waffen und Rüstungsgütern. Nimmt man die vorhandenen nationalen Daten über Genehmigungen von grenzüberschreitenden Rüstungsgeschäften und den tatsächlichen Exporten, dann erscheint der internationale Rüstungshandel in einem anderen Bild. Bei SIPRI werden nationale Regierungsangaben $\mathrm{zu}$ Rüstungsexporten und Genehmigungen erfasst und aufgelistet. Diese standen viele Jahre lang unverbunden zu den Schätzungen über Großwaffentransfers. Erst in den vergangenen Jahren schenkt SIPRI diesen Daten offenbar mehr Beachtung und beginnt damit, seine Berechnungen $\mathrm{zu}$ revidieren. In diesem Zusammenhang kam es - zumindest statistisch gesehen - zu einer enormen Erhöhung des von SIPRI geschätzten realen weltweiten Volumens an Waffenexporten und
Transfers von sonstigen Rüstungsgütern. Während SIPRI auf der Basis seiner Erfassung nationaler Angaben für das Jahr 2012 den Umfang des globalen Rüstungsmarktes noch auf 43 Milliarden US-Dollar schätzte, legte es für 2014 eine Schätzung der Höhe des globalen Rüstungsmarktes vor, die bei 94 Milliarden US-Dollar als Minimalbetrag lag (tatsächlich dürfte die Zahl deutlich höher liegen). ${ }^{18}$ Das bedeutet, dass SIPRI heute das reale Volumen des internationalen Handels mit Waffen und sonstigen Rüstungsgütern auf etwa 100 Milliarden Euro einschätzt. Diese Summe wirkt gewaltig, stellt aber gerade mal 8,5 Promille des weltweiten Handels mit Gütern dar. ${ }^{19}$ Eine weitere Aufgliederung des internationalen Waffenhandels nach schweren Waffen, leichten Waffen, Kleinwaffen und Komponenten sowie sonstigen Rüstungsgütern nimmt SIPRI nicht vor. Die US-Regierung schätzt den Gesamtumfang des gesamten Waffenhandels weltweit deutlich geringer ein. Im Jahr 2014 soll dieser bei 54 Milliarden US-Dollar gelegen haben, 2015 bei 46,2 Milliarden US-Dollar. ${ }^{20}$ Das wären dann rund fünf Promille des Weltexportes von Gütern.

Der Vergleich unter Einbeziehung nationaler Angaben lässt deutlich werden, wie sehr die Statistiken von SIPRI und der US-amerikanischen Regierung die wahren Verhältnisse bei den Rüstungsmärkten verzerren. Zwar sind die unterschiedlichen nationalen Angaben nicht unmittelbar vergleichbar, aber innerhalb der Europäischen Union gelten weitgehend die gleichen Regularien für deren statistische Erfassung und von daher ist eine hohe Vergleichbarkeit unter den EU-Staaten gegeben. Diese Daten können helfen, die Rolle Deutschlands in den internationalen Rüstungsmärkten relativ gesehen zu seinen europäischen Nachbarn klarer zu erschließen. Für den Zeitraum 2001 bis 2010 sind Daten verfügbar, die diesen Abgleich ermöglichen. Sie lassen erkennen, dass Deutschland als Exporteur von Kriegswaffen und sonstigen Rüstungsgütern deutlich hinter Frankreich und Großbritannien rangierte.

18 Vgl. zu den Zahlen für 2012 SIPRI/FES/Berghof Stiftung 2013, S. 10; für die Angabe für 2014 vgl. SIPRI/FES/Berghof Stiftung 2016, S. 21, s. a. https://www.sipri.org/databases/financial-value-globalarms-trade.

19 Im Jahr 2015 betrug das Volumen aller Güterexporte weltweit 1.183,91 Milliarden US-Dollar, Angaben laut Bundesministerium für Wirtschaft und Energie 2016

20 Theohary 2016, 8. 
Tab. 5: Die 15 weltweit größten und die drei größten deutschen wehrtechnischen Unternehmen (2016)

\begin{tabular}{|c|c|c|c|c|}
\hline Name und Rang & Sitzland & $\begin{array}{l}\text { Umsatz Wehrtechnik } \\
\text { in Mio. US-Dollar }\end{array}$ & $\begin{array}{l}\text { Gesamtumsatz p. a. } \\
\text { in Mio. US-Dollar }\end{array}$ & $\begin{array}{l}\text { Wehrtechni- } \\
\text { scher Anteil } \\
\text { in Prozent }\end{array}$ \\
\hline 1. Lockheed Martin & USA & 43.468 & 47.248 & 92 \\
\hline 2. Boeing & USA & 29.500 & 94.571 & 31 \\
\hline 3. BAE Systems & UK & 23.621 & 25.867 & 91 \\
\hline 4. Raytheon & USA & 23.384 & 24.069 & 93 \\
\hline 5. Northtrop Grumman & USA & 20.200 & 24.508 & 82 \\
\hline 6. General Dynamics & USA & 19.696 & 31.353 & 63 \\
\hline 7. Airbus Group & NL & 12.321 & 73.903 & 17 \\
\hline 8. L3 Technologies & USA & 8.879 & 10.511 & 84 \\
\hline 9. Leonardo (Finmeccanica) & Ital. & 8.526 & 13.322 & 64 \\
\hline 10. Thales & Frankr. & 8.362 & 16.837 & 50 \\
\hline 11. Almaz-Antey & RUS & 7.413 & 7.413 & 100 \\
\hline 12. United Technologies & USA & 6.888 & 57.400 & 12 \\
\hline 13. Huntington Ingall & USA & 6.778 & 7.068 & 96 \\
\hline 14. United Aircraft & RUS & 5.637 & 7.046 & 80 \\
\hline 15. Rolls Royce & UK & 4.741 & 18.675 & 25 \\
\hline 26. Rheinmetall & $\mathrm{D}$ & 3.270 & 5.753 & 57 \\
\hline 43. ThyssenKrupp Marine Systems & $\mathrm{D}$ & 1.696 & 1.696 & 100 \\
\hline 94. Diehl Defence Holding & $\mathrm{D}$ & 482 & 3.785 & 13 \\
\hline
\end{tabular}

Quelle: Defense news; http://people.defensenews.com/top-100/ (August 2017) sowie weitere Industrieangaben.

Tab. 6: Die Rangfolge der Rüstungsexporteure gemäß nationalen Angaben (2001-2010)

\begin{tabular}{ll}
\hline Exportnation & $\begin{array}{l}\text { Rüstungsexporte in Mio. } \\
\text { laufenden US-Dollar }\end{array}$ \\
\hline 1. USA & 127.550 \\
2. Russland & 72.466 \\
3. Großbritannien & $64.724(2001-2007)$ \\
4. Frankreich & 56.781 \\
5. Deutschland & 17.282 \\
6. Italien & 13.557 \\
7. Schweden & 12.851 \\
8. Spanien & 9.518 \\
9. Ukraine & 6.600 (ungefähr) \\
10. Südkorea & 6.055 \\
\hline
\end{tabular}

Quelle: SIPRI-Datenbank

Wie aus Tabelle 6 erkennbar, haben im Zeitraum zwischen 2001 und 2010 die USA mit Transfers in Höhe von über 127 Milliarden US-Dollar den internationalen Rüstungsexport angeführt. Dahinter rangiert Russland mit über 72 Milliarden US-Dollar, gefolgt von Großbritannien, welches Exporte in Höhe von fast 65 Milliarden US-Dollar getätigt hatte. Da die britischen Zahlen nur bis 2007 reichen (seit 2009 veröffentlicht die britische Regierung keine Angaben mehr über getätigte Rüstungsexporte), kann nur extrapoliert werden. Die britischen Rüstungs- transfers für den gesamten Zehn-Jahres-Zeitraum dürften dann tatsächlich zwischen 75 und 80 Milliarden US-Dollar gelegen haben, sodass Großbritannien Russland von Platz zwei verdrängte. Dann kommt Frankreich mit einem Transfervolumen von fast 57 Milliarden US-Dollar. Diese vier Staaten bilden eine Klasse für sich, das fünf-platzierte Deutschland liegt mit 17 Milliarden US-Dollar deutlich dahinter zurück, dicht gefolgt von Italien (mit 13,6 Milliarden US-Dollar) und Schweden mit fast 13 Milliarden USDollar.

Die Angaben in Tabelle 6 müssen aber relativiert werden. Auch wenn die Angaben der EU-Mitgliedstaaten in vergleichbarer Weise erstellt werden, sind nicht alle Daten in der Tabelle enthalten. Die Bundesregierung gibt lediglich Daten zu den tatsächlich erfolgten Exporten von Kriegswaffen bekannt, aber nicht zu den tatsächlich erfolgten Exporten sonstiger Rüstungsgüter. Die 17,282 Mrd. € umfassenden deutschen Exporte der Jahre 2001 bis 2016 reflektieren also nur Exporte von Kriegswaffen. Wollte man eine Vorstellung von der Höhe der deutschen Rüstungsexporte gewinnen (verstanden als Exporte von Kriegswaffen und sonstigen Rüstungsgütern), so muss ein entsprechender Wert extrapoliert werden. Eine präzise Zahl ist nicht zu ermitteln, aber man kann davon ausgehen, dass der Umfang sonstiger Rüstungsexporte unter dem Wert der Exporte von Kriegswaffenexporten liegt, 
höchstens könnte eine Verdoppelung des Exportvolumens angenommen werden. ${ }^{21}$

Eine weitere Relativierung der Daten ergibt sich aus der Tatsache, dass ein wesentlicher Teil (30 bis 60 Prozent) der von der US-amerikanischen, der britischen, der französischen, der deutschen und der italienischen Regierung gemeldeten Daten zu Rüstungstransfers (oder deren Genehmigung) Exporte im Rahmen transnationaler Rüstungsproduktionen reflektieren, also zum Großteil Exporte von Komponenten, die hin und her getätigt worden sind und die oft innerhalb einer Firma stattfanden. Von daher dürfte das eigentliche Transfervolumen dieser Rüstungsproduzenten (im Sinne des Transfers militärischer Fähigkeiten) im Vergleich zu Russland (aber auch zu Schweden) deutlich kleiner anzusetzen sein, wenn man den transnationalen Teil herausrechnet. Dies ist allerdings nicht einfach, weil es zu wenige belastbare Daten dafür gibt. Im Fall Deutschlands kann man zumindest eine Vorstellung von der Größenordnung dieses Anteils bekommen, wenn man den Anteil der sogenannten Sammelgenehmigungen am Gesamtumfang der erteilten Rüstungsexportgenehmigungen zum Ausgang nimmt. Dieser betrug im Zeitraum 2006-2016 im Durchschnitt knapp 37 Prozent. ${ }^{22}$ Ähnliche Berechnungen kommen für Großbritannien auf eine vergleichbare Größenordnung. ${ }^{23}$ Dadurch, dass eine Vielzahl von Transfers innerhalb der Firma BAE Systems stattfand, dürfte dieser Anteil vermutlich deutlich höher sein als für Deutschland. Durch eine derartige Berechnung würden die effektiven Transfers militärischer Kapazitäten aller vier europäischer Staaten und auch der USA abnehmen, es würde sich aber am Rang Deutschlands im Vergleich zu Frankreich und Großbritannien nichts ändern.

\footnotetext{
21 Da die Bundesregierung bei den Exportgenehmigungen eines Jahres eine Differenzierung nach einzelnen Kategorien vornimmt, lässt sich aus diesen Angaben zumindest eine Größenordnung des Verhältnisses zwischen Kriegswaffen einerseits und anderen Rüstungsgütern andererseits im Genehmigungsverfahren grob abschätzen. Die Angaben des Bundesministeriums für Wirtschaft und Energie (2017, 22) lassen erkennen, dass Ketten- und Radfahrzeuge, Bomben, Torpedos und Flugkörper, Kriegsschiffe und Munition (also weitgehend Kriegswaffen) weit mehr als $60 \%$ des Genehmigungsvolumens ausmachten. Das gibt eine Vorstellung von der Relation zwischen Waffenexporten zu sonstigen Rüstungsexporten. Die zurückliegenden Berichte des Bundesministeriums für Wirtschaft und Technologie kommen zu ähnlichen Einschätzungen. Ob sich diese Relation bei den tatsächlich erfolgten Exporten widerspiegelt, lässt sich nicht präzise ermitteln, da ein erheblicher Teil der Genehmigungen nicht zu Exporten führt.

22 Berechnet anhand der Daten des Bundesministeriums für Wirtschaft und Energie 2017, 29.

23 Dowdall 2004.
}

Diese Rangfolge dürfte auch Bestand haben, wenn man in Zeiten zurückgeht, in denen die transnationale Dimension der Rüstungsproduktion noch nicht so ausgeprägt war wie heute. Schon in den 1980er-Jahren lagen die von SIPRI errechneten Daten zu Großwaffenexporten weit entfernt von dem, was die nationalen Angaben westeuropäischer Staaten hergaben. Legte man die damals bekannten nationalen Angaben zugrunde, dann lag die Bundesrepublik Deutschland deutlich hinter Großbritannien und Frankreich auf Platz 5 oder 6 der internationalen Statistik. ${ }^{24}$ Diese Rangfolge macht Sinn, weil sie die unterschiedliche Größenordnung der jeweiligen wehrtechnischen Sektoren in den drei genannten europäischen Ländern ebenso widerspiegelt wie die unterschiedlichen sicherheitspolitischen Ambitionen und Bindungen. ${ }^{25}$

Zusammenfassend lässt sich feststellen, dass Deutschland im Bereich der Exporte von Großwaffensystemen und deren Hauptkomponenten zwar einer der großen Akteure ist (und das ist weitgehend den Exporten der sehr teuren Kriegsschiffe sowie auch den Exporten von gepanzerten Fahrzeugen und von Antriebsaggregaten geschuldet), aber die Behauptung, dass Deutschland mehr oder weniger dauerhaft der drittgrößte Waffenexporteur der Welt sei, hält einer kritischen Prüfung der Datenlage nicht stand und ist daher als irreführend zu qualifizieren. Im Vergleich zu den USA, Russland, Frankreich und Großbritannien dürfte Deutschland mit Abstand die Nummer fünf sein - und zwar schon für längere Zeit, nicht erst seit 2012. Rechnet man noch China hinzu (welches keinerlei nationale Angaben vorlegt, aber zunehmend als Waffenexporteur präsent ist), dann wird Deutschland von diesem inzwischen auch schon deutlich überholt worden sein. Somit dürfte es unter den weltweiten Rüstungsexporteuren eher Platz 6 einnehmen.

\section{Ist Deutschland weltweit der zweitgrößte Exporteur von Kleinwaffen?}

Kleinwaffen und leichte Waffen gelten mit Blick auf die vielen Bürgerkriege oder irregulären Kriege (an denen vor allem Milizen oder bewaffnete Banden teilnehmen)

24 Näheres bei Krause 1985.

25 Sowohl Großbritannien als auch Frankreich unterhalten aus historischer Verbundenheit zu ihren früheren Kolonien eine Reihe von Militärhilfebeziehungen und haben teilweise auch Sicherheitsgarantien übernommen. Vergleichbare Beziehungen Deutschlands gibt es nicht. 
heute als eine Art von Massenvernichtungswaffe. Das ist in dieser Allgemeinheit nicht korrekt, aber es ist wahr, dass die blutigsten Konflikte der vergangenen Jahrzehnte in Afrika, im Mittleren Osten, in Südasien und Lateinamerika zu einem Großteil mit Kleinwaffen und leichten Waffen ausgetragen worden sind. Was hierbei leider übersehen wird, ist, dass natürlich auch Großwaffensysteme (insbesondere Artillerie, Mehrfachraketenwerfer, Kampfhubschrauber und Bombenflugzeuge) keinesfalls eine vernachlässigbare Größe darstellen. Tatsächlich sind sie die wichtigsten Kampfkraftverstärker bei regional beschränkten Konflikten und Bürgerkriegen. Es gibt allerdings seit einigen Jahrzehnten kaum mehr klassische Kriege, bei denen große Heere, Luftstreitkräfte und Seestreitkräfte zusammenwirken und bei denen Großwaffensysteme optimal zum Einsatz kommen. Von daher ist die Frage schon von Bedeutung, ob deutsche Rüstungsexporte im Bereich der Kleinwaffen eine wesentliche Rolle spielen.

Dabei muss vorausgeschickt werden: Es gibt unterschiedliche Definitionen von Kleinwaffen, außerdem muss zwischen diesen und leichten Waffen unterschieden werden. Gemäß den in Deutschland und der EU geltenden Regeln werden unter Kleinwaffen nur die folgenden Kategorien von Waffen verstanden: Gewehre mit Kriegswaffenlistennummer (das heißt Gewehre, die für den Einsatz in militärischen Auseinandersetzungen hergestellt werden, insbesondere Sturmgewehre), Maschinenpistolen, Maschinengewehre sowie Waffen für hülsenlose Munition. Nach deutscher Klassifikation sind Kleinwaffen eine Untergruppe der Handfeuerwaffen, zu denen auch Sport-, Jagd-, Polizei- und Waffen zur Selbstverteidigung gehören. Wissenschaftliche Einrichtungen wie das Small-Arms-Survey-Projekt in Genf oder die Conflict Armaments Research Group in London fassen unter dem Begriff der Kleinwaffen neben Handfeuerwaffen (firearms) und den für Kriegszwecke gebrauchten Gewehren auch leichte Waffen, das sind weitere Infanteriewaffen wie Mörser, Panzerfäuste, andere Panzerabwehrmittel, tragbare Luftabwehrraketen, Handgranaten, leichte Flak und Ähnliches.

Rüstungskritiker behaupten, dass Deutschland einer der führenden Exporteure von Kleinwaffen sei (angeblich der zweitgrößte nach den USA) und dass alleine diese Tatsache belege, dass es in Deutschland nur um Profit gehe und in der Politik niemand daran interessiert sei, was mit all den Waffen geschehe. Belastbare Zahlen gibt es nur sehr wenige und diejenigen, die vorhanden sind, lassen auf den ersten Blick tatsächlich den Eindruck entstehen, dass Deutschland unter den ganz großen Exporteuren zu finden ist. Das in Genf beheimatete Forschungsprojekt Small Arms Survey veröffentlicht seit mehreren Jahren
Daten über den geschätzten Umfang von internationalen Transfers von Kleinwaffen (im Sinne der oben genannten breiteren Definition) sowie von Feuerwaffen. Und dort steht Deutschland tatsächlich ganz oben in der Statistik.

Das Small Arms Survey basiert seine Daten auf den Erhebungen der Vereinten Nationen, die jährlich eigene Statistiken über alle Handelstransfers veröffentlichen (United Nations Commodity Trade Statistics Database, abgekürzt UN Comtrade). Seitdem es ein UN-Waffenregister gibt und dort Staaten ihre Exporte von Großwaffensystemen, leichten Waffen und Kleinwaffen angeben, fügt Comtrade diese in die allgemeinen Handelsstatistiken ein. Auf der Basis dieser Daten wird eine grobe Einschätzung des internationalen Handels mit Kleinwaffen und leichten Waffen vorgenommen. Für das Jahr 2012 stellten die Genfer Experten ein globales Volumen an Klein- und Leichtwaffenexporten in Höhe von knapp über 5 Milliarden US-Dollar fest, was gegenüber 2001 fast eine Verdoppelung darstelle. ${ }^{26}$ Im Jahre 2013 habe sich das Volumen auf 5,8 Milliarden und im Jahr 2014 auf mehr als 6 Milliarden US-Dollar erhöht. ${ }^{27}$

Bezogen auf das vergangene Jahrzehnt würden sich die Hauptexporteure in mehrere Klassen einteilen lassen. $\mathrm{Zu}$ den Top-Exporteuren mit für über 500 Millionen USDollar exportierten Kleinwaffen pro Jahr hätten die USA, Italien und Deutschland (in dieser Reihenfolge) gezählt. Allerdings habe im Jahr 2010 die Bundesrepublik den Platz zwei vor Italien eingenommen ${ }^{28}$ - seither geistert die Behauptung durch die deutsche rüstungskritische Debatte, dass Deutschland weltweit der zweitgrößte Exporteur von Kleinwaffen sei. Die zweite Gruppe, die der größeren Exporteure, umfasse 13 Länder, das seien nacheinander Brasilien, Österreich, Südkorea, die Türkei, Russland, Tschechien, Israel, Belgien, Kroatien, China, die Schweiz, Japan und Spanien. Sie würden Kleinwaffen im Wert zwischen 100 und 500 Millionen US-Dollar pro Jahr exportieren. Ihnen folge eine Gruppe von relevanten Exporteuren, die für weniger als 100 Millionen US-Dollar im Jahr exportiere. ${ }^{29}$ Die oben genannte Reihenfolge der führenden fünf Exporteure von Klein- und Leichtwaffen habe sich seit 2001 kaum geändert (USA, Italien, Deutschland, Brasilien, Österreich). ${ }^{30}$ Allerdings

\footnotetext{
26 Small Arms Survey 2015, 88.

27 Pavesi 2016, 14; Holton/Pavesi 2017, 14.

28 Small Arms Survey 2013, 180.

29 Pavesi 2016, S. 23.

30 Small Arms Survey 2015, S. 87.
} 
habe im Jahr 2014 Brasilien Deutschland vom dritten Platz verdrängt. “31

Schaut man sich die Daten und die damit einhergehenden Erläuterungen genauer an, so wird allerdings schnell erkennbar, dass die Aussage, Deutschland beliefere an führender Stelle die Krisenzonen der Welt mit Kleinwaffen, daraus nicht abgeleitet werden kann. Eher ist das Gegenteil der Fall:

- Zum einen meldet nur eine Minderheit der Staaten die erforderlichen nationalen Daten an das Transferregister der Vereinten Nationen. ${ }^{32}$ Bei vielen sind die überlieferten Daten zudem unvollständig oder Kleinwaffentransfers werden gar nicht gemeldet. Dies trifft besonders für China, den Iran und Russland zu, deren Kleinwaffen und leichte Waffen heute in großen Mengen bei nahezu allen Kleinkriegen auftauchen. Die führende Position der USA, Italiens und Deutschlands beim internationalen Handel mit Klein- und Leichtwaffen reflektiert also erst einmal nur die Tatsache, dass es sich bei ihnen um westliche Demokratien und Rechtsstaaten handelt, die Transparenz herstellen wollen und können und die sich an international gemachte Zusagen halten. Die Bundesregierung wird vom Small Arms Survey ausdrücklich für die Offenheit und Gewissenhaftigkeit gelobt, mit der sie ihre Daten präsentiert.

- Zum anderen lassen die Daten erkennen, dass es sich bei den deutschen Transfers von Kleinwaffen zum weitaus überwiegenden Teil um neue Sport-, Jagdund Polizeiwaffen handelt. Empfänger sind überwiegend westliche Staaten. Transfers an Länder in Afrika, Asien und Lateinamerika kommen vor, stellen aber mengenmäßig eine nachgeordnete Rolle dar. Oft sind es dann Lieferungen von gebrauchten Waffen an VN-Missionen in diesen Ländern oder es handelt sich - wie im Fall der Lieferung von Waffen an die Peschmerga-Milizen im Nordirak - um Transfers, die nach einer sorgfältigen Abwägung der Vor- und Nachteile erfolgt sind. In anderen Fällen handelt es sich um Länder, zu denen die Bundesregierung aus außenpolitischen Gründen gute Beziehungen unterhält und bei denen Kleinwaffenexporte als vertretbar und kontrollierbar angesehen werden (Saudi-Arabien, Vereinigte Arabische Emirate, Katar). Die deutschen Kleinwaffenexporte gingen im Zeitraum zwischen 2001 und 2012 überwiegend in die USA (1,25 Milliarden USDollar), nach Frankreich (380 Millionen US-Dollar), in die Schweiz (250 Millionen US-Dollar), nach Österreich (220 Millionen US-Dollar) und nach Norwegen (195 Millionen US-Dollar) - allesamt keine Staaten, in denen Bürger- und Sezessionskriege toben. ${ }^{33}$ Und die Exporte bestanden überwiegend aus Handfeuerwaffen, die keine Kriegswaffen waren. Exporte von deutschen Kleinwaffen (im Sinne von Kriegswaffen) fallen im Vergleich dazu gering aus. Zwar gibt es keine Zahlen über die tatsächlich getätigten Transfers, aber die Daten über erteilte Genehmigungen sind schon aussagekräftig, weil sie die Größenordnungen erkennen lassen. Im Jahr 2016 betrug der Wert aller erteilten Exportgenehmigungen für Kleinwaffen aus Deutschland (also Kriegswaffen) knapp 47 Millionen Euro. Davon entfielen auf den EU-Raum knapp 28 Millionen Euro, auf NATO-Mitglieder beziehungsweise gleichgestellte Staaten etwa 2,5 Millionen Euro und auf Drittländer ungefähr 16,4 Millionen Euro. ${ }^{34}$ Diese Zahlen sind keine einmaligen Werte für ein bestimmtes Jahr, sondern markieren einen Trend, der über zehn Jahre anhält (Tabelle 7). Sie lassen eines erkennen: Für die Versorgung der zahlreichen Konfliktherde und Bürgerkriege in Afrika, im Nahen und Mittleren Osten, in Süd-, Zentral- und Ostasien sowie Lateinamerika mit Gewehren, Sturmgewehren und Maschinengewehren spielt Deutschland keine Rolle. Sofern es zu Transfers kommt, bleiben diese mengenmäßig begrenzt. In den vergangenen zehn Jahren lagen die Genehmigungen von kleinen Kriegswaffen für Drittländer zwischen 14 und 42 Millionen Euro pro Jahr, wobei das Volumen der tatsächlich getätigten Exporte daruntergelegen haben dürfte. Allerdings muss ergänzt werden, dass Exporte von Infanteriewaffen, die nicht unter die deutsche beziehungsweise europäische Definition von Kleinwaffen fallen, in dieser Zahl nicht berücksichtig sind.
31 Holton/Pavesi 2017, 17

32 Auswärtiges Amt 2017, S. 38.
33 Small Arms Survey 2015.

34 Bundesministerium für Wirtschaft und Energie 2017, S. 30. 
Tab. 7: Einzelgenehmigungen für deutsche Kleinwaffen 2006-2016 (in Millionen Euro)

\begin{tabular}{lrrll}
\hline Jahr & EU-Länder & $\begin{array}{l}\text { NATO oder } \\
\text { gleichgestellte } \\
\text { Länder (ohne EU) }\end{array}$ & Drittländer & Summe \\
\hline \multicolumn{5}{c}{} \\
\hline 2006 & 11,45 & 10,23 & 15,6 & 37,28 \\
2007 & 9,35 & 9,38 & 30,2 & 48,93 \\
2008 & 22,72 & 28,94 & 17,18 & 68,85 \\
2009 & 35,97 & 20,10 & 14,32 & 70,40 \\
2010 & 19,42 & 13,81 & 16,30 & 49,54 \\
2011 & 10,03 & 9,95 & 17,92 & 37,90 \\
2012 & 12,84 & 26,22 & 37,09 & 76,15 \\
2013 & 6,80 & 33,59 & 42,23 & 82,63 \\
2014 & 6,23 & 19,57 & 21,63 & 47,43 \\
2015 & 11,13 & 6,81 & 14,49 & 32,43 \\
2016 & 27,96 & 2,55 & 16,38 & 46,89 \\
\hline
\end{tabular}

Quelle: Bundesministerium für Wirtschaft und Energie 2017, 30.

Wie groß ist der Anteil deutscher Exporte von Kleinwaffen und leichten Waffen im globalen Vergleich? Diese Frage ist schwer zu beantworten, denn es gibt keine verlässliche Schätzung des jährlichen Niveaus des Transfers von kleinen Kriegswaffen (einschließlich Infanteriewaffen) weltweit oder in Krisenregionen. Die vom Small Arms Survey genannten Schätzwerte in der Größenordnung von 5 bis 6 Milliarden US-Dollar sind irreführend, weil sie primär den innerwestlichen Handel mit Polizei-, Sportund Jagdwaffen widerspiegeln. Die eigentlich problematischen Transfers in Krisengebiete kommen in dieser Statistik weitgehend nicht vor. Es ist eher davon auszugehen, dass der globale Handel mit Kleinwaffen und leichten Waffen pro Jahr in einer Größenordnung zwischen 10 bis 20 Milliarden US-Dollar anzusetzen ist. Aber das ist nicht mehr als eine grobe Schätzung ausgehend von der Annahme, dass die Vermutung von SIPRI zutrifft, wonach der weltweite Handel mit Waffen und Rüstungsgütern ein Niveau von etwa 100 Milliarden US-Dollar hat.

Ein nicht unwesentlicher Teil des Transfers von Kleinwaffen und leichten Waffen wird dabei über graue und schwarze Märkte abgewickelt. ${ }^{35}$ Es wird generell unterschieden zwischen grauen Märkten (wo Staaten undercover und oft unter Verletzung eigener Gesetze oder internationaler Sanktionsbeschlüsse Waffen an Milizen oder Regierungen liefern) und schwarzen Märkten (wo private Akteure Waffen illegal an Milizen oder Regierungen transferieren). $\mathrm{Zu}$ den bekanntesten Fällen grauer Transfers gehörten die Waffenlieferungen im Rahmen der Iran-Con-

35 Vgl. Schroeder/Lamb 2006, Marsh 2002, siehe auch Jonsson/ Brennan 2014
tra-Affäre zu Zeiten von US-Präsident Ronald Reagan ${ }^{36}$ oder die Waffenlieferungen der USA an die Mujaheddin in Afghanistan. Illegale Schwarzmarkttransfers sind vor allem in Afrika, Kolumbien sowie Südasien zu beobachten gewesen, wo sie ein wesentlicher Faktor zur Verlängerung und Verschärfung der dortigen Konflikte waren und sind. Einzelne Fälle werden immer wieder aufgedeckt, aber ein umfassendes Bild ist nicht zu gewinnen. Auffällig ist nur, dass in den vergangenen drei Jahrzehnten immer wieder Fälle bekannt wurden, in denen aus Nachfolgestaaten der Sowjetunion oder aus früheren Mitgliedstaaten des Warschauer Paktes große Waffenlieferungen per Lufttransport stattgefunden haben. ${ }^{37}$ Vor allem im Zusammenhang mit der Verhaftung und Verurteilung des russischen Waffenhändlers Victor But wurde eine Vielzahl von Details über diesen Markt enthüllt. ${ }^{38}$ Nur wenig ist bekannt über die Rolle des Internets als Verstärkungsfaktor des illegalen Handels mit Kleinwaffen. ${ }^{39}$

Was das jährliche Volumen des illegalen Handels mit Waffen betrifft, so ist man auch hier auf Schätzungen angewiesen. Die NGO Global Financial Integrity hat den Umfang des illegalen Handels (also Schwarzmarkt) mit Waffen (egal ob klein oder groß) für 2014 auf eine Größenordnung zwischen 1,7 und 3,5 Milliarden US-Dollar geschätzt. ${ }^{40}$ Diese Zahl reflektiert keine eigenen Recherchen, sondern basiert auf der Annahme, dass mindestens zehn Prozent des globalen Handels mit Waffen illegal erfolgen. ${ }^{41}$ Sie geht aus von einem globalen Niveau des Waffentransfers zwischen 17 und 35 Milliarden US-Dollar pro Jahr. Berücksichtigt man die Schätzung von SIPRI, wonach der weltweite Handel mit Waffen und sonstigen Rüstungsgütern ein Niveau von 100 Milliarden US-Dollar haben soll, dann wäre es naheliegend davon auszugehen, dass der illegale Handel mit schweren Waffen, Kleinwaffen und leichten Waffen in der Größenordnung von 10 Milliarden US-Dollar jährlich anzusetzen ist. Aber das sind alles nur Spekulationen. Nimmt man die oben genannten Zahlen der US-amerikanischen Regierung zum Ausgang, liegt der illegale Anteil bei 5 Milliarden pro Jahr.

Folgt man den Analysen von Conflict Armaments Research und Small Arms Survey, so ist davon auszugehen, dass gegenwärtig Russland, China, die USA und der Iran

36 Vgl. Walsh 1993.

37 Vgl. Schroeder/Lamb 2006, 72, Thachuk/Saunders 2014.

38 Zum Hintergrund vgl. Farah/Braun 2008; zu den Umständen seiner Verurteilung vgl. „25 Jahre für den Händler des Todes“, NZZ online, 6. 4. 2012, https://www.nzz.ch/25-jahre-fuer-den-haendlerdes-todes-1.16307701.

39 Vgl. Paoli/Aldridge/Ryan/Warnes 2017.

40 Vgl. May 2017, 14.

41 Vgl. Arsovska 2014, 296. 
sowie andere ehemalige Ostblockländer die führenden Exporteure sind. Eine klare Rangliste der Exporteure zu erstellen ist nicht möglich. Die von der Bundesregierung veröffentlichten Zahlen lassen zumindest den Schluss zu, dass der deutsche Anteil an legalen Transfers von Kleinwaffen minimal ist und Deutschland mit deutlich weniger als einem Prozent Marktanteil ganz bestimmt nicht in der Spitzengruppe der Exportstaaten zu finden sein dürfte. Am grauen Waffenmarkt hat die Bundesregierung keinen Anteil und über die Vorkommen (meist alter) deutscher Waffen an den Arsenalen von Schwarzmarkthändlern kann bestenfalls spekuliert werden.

Die Annahme einer vernachlässigbaren Bedeutung Deutschlands für den internationalen Transfer mit Kleinwaffen in Kriegs- und Krisengebiete wird auch durch Erkenntnisse des Small Arms Survey sowie des Projektes Conflict Armaments Research unterstützt. Beide Forschungsprojekte gehen davon aus, dass in den vergangenen drei Jahrzehnten die Waffenversorgung von Konfliktparteien bei regionalen Kriegen oder Bürgerkriegen bestimmte Muster hat erkennen lassen, die in keiner Weise auf eine herausragende Rolle Deutschlands schließen lassen:

- Die meisten Infanteriewaffen und schweren Waffen, die in regionalen Kriegen oder Bürgerkriegen zum Einsatz kommen, sind älteren Datums. Sie stammen zumeist aus der Zeit des Kalten Krieges und wurden entweder über Schwarzmärkte vertrieben oder entstammen den Arsenalen jener Staaten, die große Überschussmengen an derartigen Waffen haben. Deutschland ist nicht unter den 20 Staaten zu finden, die die größten Überschussmengen aufweisen (Tabelle 8). Dort stehen vorneweg vielmehr Russland, China, Vietnam und die Ukraine. ${ }^{42}$ Aber auch der Iran und der Sudan werden immer wieder als Rüstungslieferanten erwähnt. ${ }^{43}$

- Bei Gewehren, Pistolen und Maschinengewehren sowie anderen Infanteriewaffen überwiegen mittlerweile Waffen mit Kalibern, welche typisch für den früheren Warschauer Pakt beziehungsweise Russland und China sind. Besonders Sturmgewehre der Serie AK (Kalaschnikow), Panzerabwehrraketen und Mörser russischen und chinesischen Ursprungs sind in allen Konflikten in großen Mengen zu finden. ${ }^{44}$

42 Karp 2014, zum Problem südosteuropäischer Staaten mit Überschussmunition vgl. Lararevic 2010.

43 Florquin/Le Brun/Leff/Schroeder 2014.

44 Smith 2003, Pétard/Glatz 2010, Hazen/Horner 2010, Berman 2012, Bevan 2013, Florquin/Le Brun/Leff/Schroeder 2014, Inowlocki 2014.
- Viele Kleinwaffen, insbesondere aber deren Munition, werden auch in Eigenproduktion hergestellt oder gebrauchte Waffen werden modifiziert. Mehr als 60 Staaten stellen heute Kleinwaffen und leichte Waffen her. ${ }^{45}$ Viele Waffen sind zudem Beutewaffen.

- Einzeluntersuchungen zu Waffenarsenalen in Konfliktgebieten lassen erkennen, dass deutsche Infanteriewaffen oder Kleinwaffen kaum noch eine Rolle spielen. ${ }^{46}$ Auch G3-Gewehre tauchen immer weniger auf. ${ }^{47}$

Diese Trends reflektieren die Tatsache, dass die Bundesregierung in den vergangenen Jahrzehnten sehr vorsichtig mit Kleinwaffentransfers und Transfers von weiteren Infanteriewaffen in Drittstaaten umgegangen ist. Sie hat dazu Leitlinien entwickelt, die entsprechend angewandt worden sind. Mit dieser Politik sind die Konsequenzen aus dem geradezu leichtsinnigen Umgang gezogen worden, den frühere Bundesregierungen (in den 1960er-, 1970erund frühen 1980er-Jahren) mit Exporten des G3-Sturmgewehrs praktiziert hatten. Das G3-Gewehr wurde damals nicht nur in großen Mengen produziert und exportiert (in NATO-Staaten, aber auch in Dutzende von Drittländern), es wurden auch Lizenzen zur Produktion in über 15 Ländern erteilt. Dadurch wurden Sturmgewehre des Typs G3 (von denen es auch Varianten gibt) zu einem der meist produzierten Sturmgewehre der Welt. Diese Aussage muss aber in einer Hinsicht relativiert werden: Die Zahl der weltweit seit den 1950er-Jahren produzierten G3-Sturmgewehre dürfte bei 7 Millionen liegen, das ist weit weniger als die im gleichen Zeitraum produzierten 12 Millionen US-amerikanischen M16-Sturmgewehre und eine geradezu kleine Anzahl im Vergleich zu den über 100 Millionen verschiedenen Varianten der russischen Kalaschnikow (siehe Tabelle 9). ${ }^{48}$ Heute werden G3-Sturm-

45 Jenzen-Jones 2014.

46 Conflict Armaments Research 2016c, Conflict Armaments Research 2016d, Tessieres 2016, Conflict Armaments Research 2014.

47 Nur in einer Übersicht des Conflict Armaments Research tauchen neben den Waffen meist aus russischer, chinesischer oder iranischer Herkunft auch noch G3-Gewehre auf, vgl. Conflict Armaments Research 2013. Siehe auch die Analyse bei Tessières (2018, 47 f), derzufolge bei Waffenfunden im Norden Nigers nur kleine Bestände von G3 Gewehren gefunden worden seien, hingegen umfangreiche Bestände von Sturmgewehren aus Russland, China, Bulgarien, Ägypten und Polen sowie jede Menge Pistolen aus der Türkei.

48 Das hält deutsche Rüstungskritiker nicht davon ab, die Behauptung in die Welt zu setzen, das G3-Gewehr und andere Waffen von Heckler \& Koch seien die „weltweit am meisten verbreiteten Kleinwaffen“; vgl. Andreas Zumach: „Armut, Rüstungsexporte, Killerspiele, Afghanistankrieg - warum die Überwindung der Gewalt so schwer fällt”, Rede gehalten beim Festakt der Evangelischen Kirche 
gewehre nicht mehr hergestellt (lediglich noch in schlechter Qualität in Pakistan) und dienen in vielen regulären Streitkräften nur noch als Reserve. Aber sie sind noch auf Schwarzmärkten erhältlich. Wie groß der Anteil der G3-Gewehre am Volumen der Schwarzmärkte ist, lässt sich nicht bestimmen. Es lässt sich bestenfalls eine ungefähre Vorstellung davon gewinnen, wenn man sich die Produktionszahlen der wichtigsten Sturmgewehre und ihrer Varianten vor Augen hält. Demnach könnten theoretisch bis zu 5 Prozent aller Sturmgewehre auf den internationalen Schwarzmärkten aus der G3-Familie stammen (siehe Tabelle 9). Aber diese Zahl müsste eher nach unten korrigiert werden. In dem Maße, in dem bei regionalen Kriegen oder Bürgerkriegen die Kaliber aus dem Warschauer Pakt dominierend geworden sind, dürften G3-Gewehre bevorzugt ausgemustert worden sein.

Auch bei tragbaren Luftabwehrraketen, die derzeit eine der effektivsten Waffen bei Aufständen oder Guerillakriegen sind und die ein hohes Potenzial für terroristische Anschläge darstellen, gibt es keine deutsche Beteiligung. Hier dominieren eindeutig Produkte sowjetischer/russischer und chinesischer Bauart. ${ }^{49}$ Das gleiche Bild bietet sich bei Mörsern, Maschinengewehren, Handgranaten etc. Lediglich die in deutsch-französischer Kooperation entwickelten Panzerabwehrraketen des Typs MILAN finden sich in mehreren Arsenalen.

Zusammengenommen ist die Behauptung, Deutschland sei einer der weltweit größten Lieferanten von Kleinwaffen für Konflikte in Afrika, dem Nahen und Mittleren Osten, Zentral-, Süd- und Ostasien oder Lateinamerika, mit nichts zu belegen. Sie ist offenkundig falsch, eine fake news. Die Hartnäckigkeit, mit der sich diese Behauptung in den Medien, der Politik und auch der wissenschaftlichen Debatte hält, ist daher umso erstaunlicher. Man gewinnt den Eindruck, dass Zahlen, die dem Eindruck einer herausragenden deutschen Rolle bei Waffenexporten widersprechen, schlichtweg nicht in ein vorgefasstes Bild passen, welches sich Medien und Politiker machen. In diesem Feld herrscht eine kognitive Dissonanz vor, die für eine aufgeklärte Demokratie erschreckend ist.

in Hessen und Nassau (EKHN) zum Reformationsfest am 31. 10. 2010 in der Lutherkirche in Wiesbaden, http://www.lebenshaus-alb.de/ magazin/006579.html.

49 Schroeder 2013, Rigual 2013, Rigual 2014, Tarr 2015.
Tab. 8: Überschußmengen an Klein- und Infanteriewaffen - die zwanzig größten Arsenale

\begin{tabular}{lc}
\hline Land & Zahl der Kleinwaffen in Mio \\
\hline Russland & 26 \\
VR China & 21 \\
Vietnam & 8 \\
Ukraine & 7 \\
Nordkorea & 6,2 \\
Südkorea & 4,7 \\
USA & 2,7 \\
Indien & 2,6 \\
Taiwan & 2,6 \\
Iran & 2,5 \\
Türkei & 2,5 \\
Pakistan & 2,0 \\
Polen & 2,0 \\
Spanien & 1,8 \\
Ägypten & 1,6 \\
Italien & 1,6 \\
Brasilien & 1,3 \\
Frankreich & 1,3 \\
Indonesien & 0,9 \\
Großbritannien & 0,9 \\
\hline
\end{tabular}

Quelle: Karp 2013

Tab. 9: Die wichtigsten Sturmgewehre und ihre Produktion seit dem Zweiten Weltkrieg

\begin{tabular}{|c|c|c|c|c|}
\hline Typ & Hersteller & $\begin{array}{l}\text { Lizenz- } \\
\text { produktion }\end{array}$ & $\begin{array}{l}\text { Bislang } \\
\text { hergestellt, } \\
\text { in Mio }\end{array}$ & $\begin{array}{l}\text { Im Umlauf, } \\
\text { in Mio }\end{array}$ \\
\hline $\begin{array}{l}\text { Kalaschni- } \\
\text { kow (AK), } \\
\text { unter- } \\
\text { schiedliche } \\
\text { Varianten }\end{array}$ & $\begin{array}{l}\text { Ischmasch, } \\
\text { Russland }\end{array}$ & $\begin{array}{l}\text { In Dut- } \\
\text { zenden } \\
\text { Ländern, } \\
\text { darunter } \\
\text { eigene } \\
\text { Weiterent- } \\
\text { wicklungen }\end{array}$ & Ca. 100 & Ca. $40-60$ \\
\hline M 16 & $\begin{array}{l}\text { Eugene } \\
\text { Stoner, Colt } \\
\text { Defense LLC, } \\
\text { USA }\end{array}$ & $\begin{array}{l}\text { In mehreren } \\
\text { Ländern }\end{array}$ & Ca. 12 & Ca. $6-8$ \\
\hline G3 & $\begin{array}{l}\text { Heckler } \\
\text { \& Koch, } \\
\text { Deutschland }\end{array}$ & $\begin{array}{l}\text { In } 15 \\
\text { Ländern }\end{array}$ & Ca. 7 & Ca. 3 \\
\hline FN FAL & $\begin{array}{l}\text { Fabrique } \\
\text { Nationale } \\
\text { Herstal, } \\
\text { Belgien }\end{array}$ & & Ca. 5,5 & Ca. 3-4 \\
\hline
\end{tabular}

Quellen: Kilicoat 2007, Bevan 2013, Bevan 2014, Jenzen-Jones 2015; zu den Angaben zu den AK Kalashnikows und Nachfolgern vgl. RIA Novosti vom 22. 10. 2012 


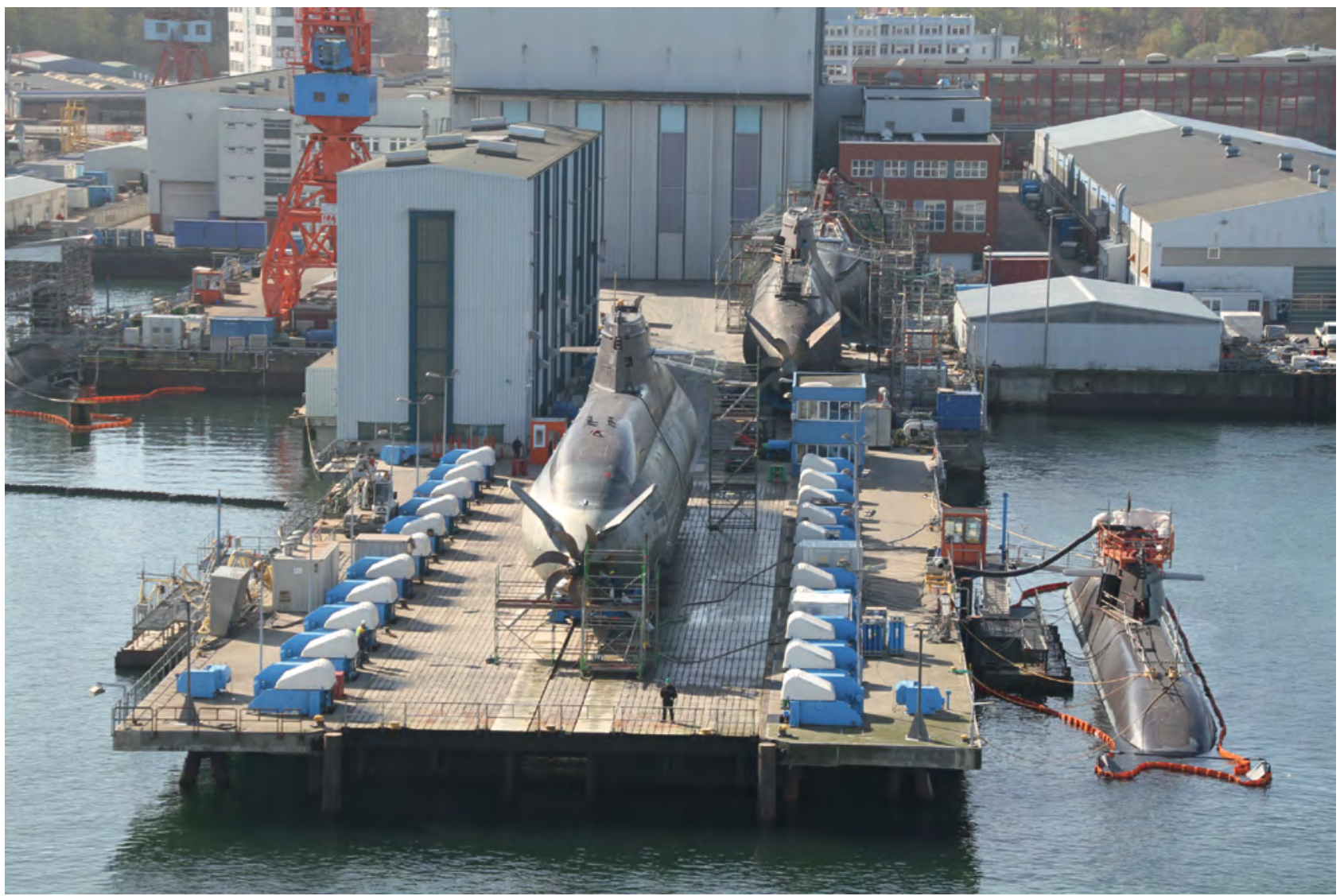

Deutsche Rüstungsexporte sind zum Großteil Exporte von Kriegsschiffen - Blick auf Thyssen-Krupp-Marine Systems in Kiel, Nachweis: Common Wikipedia, Foto: Bjoertvedt

\section{Welche Konsequenzen haben deutsche Rüstungsexporte?}

In der rüstungskritischen Literatur, aber auch in deutschen Medien, wird in der Regel behauptet, dass infolge deutscher Rüstungsexporte regionale oder innerstaatliche Konflikte zu Kriegen eskalieren würden. Besonders gerne wird darauf verwiesen, dass bei Konflikten beide Seiten mit deutschen Waffen kämpften. Liest man die entsprechenden Bücher, Artikel oder Pamphlete, gewinnt man den Eindruck, deutsche Waffen würden eigentlich bei allen größeren Konflikten eine zentrale Rolle spielen. Neuerdings werden sie sogar mit Kindersoldaten in Verbindung gebracht. ${ }^{50}$ Derartige Publikationen beginnen meistens mit der Verbreitung des G3-Gewehrs und erwähnen dann weitere deutsche Waffen oder Systeme, die bei Konfliktparteien gefunden worden sind. Aus der Aneinanderreihung derartiger Einzelinformationen entsteht dann ein Bild, demzufolge überall in der Welt an erster Stelle mit deutschen Waffen gekämpft wird. Das lädt dann ein zu munteren Spekulationen. Der deutsche Rüstungskritiker Jürgen Grässlin etwa vermutete, dass allein durch das G3-Gewehr und andere Waffen von Heckler \& Koch seit den 1960er-Jahren über zwei Millionen Menschen getötet worden seien. ${ }^{51}$ Am häufigsten wird dabei die Rolle deutscher Waffenexporte für die Eskalation des Bürgerkrieges im Sudan thematisiert. Auch Libyen sowie Syrien, Mexiko und Kolumbien werden als schlimme Beispiele für die Folgen deutscher Rüstungsexporte genannt.

Solche Behauptungen beruhen auf der Zusammenstellung einzelner Meldungen über das Auftauchen deutscher Waffen in einer Konfliktzone und sind methodisch unseriös. Sie vermitteln keinen verlässlichen Einblick in das tatsächliche Kriegsgeschehen in den betroffenen Regionen und Ländern und lassen auch keine Beurteilung der Bedeutung zu, die Waffen aus deutscher Produktion für die Kämpfe oder für die Konfliktdynamik haben. Die Schätz- 
werte über Todesopfer beruhen auf teilweise grotesken Verzerrungen und lassen sich im Vergleich mit den Statistiken etablierter Forschungsinstitutionen und internationaler Organisationen relativ schnell widerlegen..$^{52}$ Will man eine belastbare Einschätzung der Folgen deutscher Rüstungstransfers für einzelne Konfliktzonen vornehmen, so muss man eine differenzierte Konfliktanalyse an den Anfang stellen, die die Eskalationsdynamik beschreibt und man muss vor allem Analysen heranziehen, die die Bewaffnung der Konfliktparteien in toto abbilden und nicht nur auf deutsche Waffen schauen. Die folgenden Beispiele zeigen, dass die in der rüstungskritischen Literatur aufgestellten Behauptungen einer empirischen Überprüfung nicht standhalten.

52 Die oben genannte Schätzung von Grässlin fällt schon bei einem einfachen Plausibilitätstest in sich zusammen. Er geht von einer Schätzung des Internationalen Roten Kreuzes aus den 1990er-Jahren aus, der zufolge bei 41 analysierten Konfliktgebieten im Schnitt 63 Prozent der Todesopfer durch Gewehreinwirkungen ums Leben kamen. Wenn seit 1961 rund 30 Millionen Menschen in Kriegen gestorben sind, dann müssten seiner Rechnung zufolge davon 18,9 Millionen durch Gewehre ums Leben gekommen sein. Bei einem Anteil von 8 Prozent von Heckler \& Koch Gewehren am Markt für Sturmgewehre weltweit würde das bedeuten, dass 1,5 Millionen Menschen allein durch Gewehre dieser Firma getötet worden seien. Später revidierte er die Schätzung des Marktanteils der Gewehre von Heckler \& Koch auf phantastische 11 Prozent, was einer Zahl von 15 Millionen Gewehren entspräche (eine Größenordnung, die weit jenseits dessen liegt, was die Firma herstellen kann). Damit erhöhte Grässlin die Zahl der Toten gleich auf 2,1 Millionen. Auch schreibt er, dass im Durchschnitt alle 14 Minuten ein Mensch infolge der Einwirkung einer Waffe von Heckler \& Koch stirbt. Das sind recht merkwürdige, um nicht zu sagen groteske Berechnungen. Der Marktanteil aller Heckler\&-Koch-Waffen an den seit 1950 produzierten Gewehren (vor allem Sturmgewehren) liegt global gesehen durchaus in einer Größenordnung von 5 bis 6 Prozent, was hauptsächlich an der weiten Verbreitung des G3-Gewehres liegt, welches allerdings immer mehr aus Arsenalen ausgemustert wird. Aber aus dieser Verteilung kann man nicht notwendigerweise schließen, dass diese Waffen ständig in Kriegen eingesetzt werden. Wie oben gezeigt wird, lassen die Erkenntnisse über die in heutigen Kriegen verwandten Waffen diesen Schluss nicht zu. Waffen deutscher Provenienz spielen allenfalls noch eine Nebenrolle in diesen Kriegen. Auch die von Grässlin behauptete Annahme, wonach alle 14 Minuten eine Heckler-\&-Koch-Waffe einen Menschen töte, ist absurd. Das würde bedeuten, dass weltweit jährlich etwa 38.000 Menschen durch Heckler-\&-Koch-Gewehre ums Leben kommen. Nach Schätzungen des IISS bewegte sich in den vergangenen Jahren die Zahl der globalen Konfliktopfer pro Jahr zwischen 150.000 und 170.000 (worin auch die Opfer von Bürgerkriegen und auch die Opfer des mexikanischen Drogenkrieges mitgezählt sind, vgl. IISS 2017b, 5). Dann müsste etwa ein Viertel aller Kriegstoten weltweit jährlich durch Heckler-\&-Koch-Gewehre verursacht worden sein. Das ist ziemlich unwahrscheinlich, wenn man die durchgehende Dominanz russischer und chinesischer Infanteriewaffen bei fast allen heutigen bewaffneten Konflikten berücksichtigt.

\subsection{Fallbeispiel Sudan}

Der Sudan ist für viele Rüstungskritiker ein Musterfall der verhängnisvollen Wirkung deutscher Waffen. Tatsächlich hat der Sudan in den 1960er-Jahren 30.000 Stück deutscher G3-Gewehre (sowie MP5-Maschinenpistolen und HK21-Maschinengewehre) bezogen und eine dazu passende Munitionsfabrik erworben. Das G3-Gewehr wurde damals zum Standard-Sturmgewehr der neu gebildeten sudanesischen Streitkräfte. Wie oben bereits erwähnt, gibt es bezüglich der Fehler deutscher Politik beim freizügigen Export von G3-Gewehren in über 80 Länder (darunter viele Drittländer) und bei der Erteilung von Lizenzen zu deren Eigenproduktion in über 15 Ländern (darunter Iran und Pakistan) nichts zu entschuldigen. Aber kann man die Militarisierung des Sudans und insbesondere die Eskalation des in der Mitte der 1980er-Jahre ausgebrochenen Bürgerkrieges im Sudan primär auf diese und andere Waffen von Heckler \& Koch zurückführen? Zumindest argumentieren so deutsche und britische Journalisten, die sich auf Studien von Friedens- und Konfliktforschern berufen. So schrieb der britische Guardian unter Berufung auf nicht näher genannte wissenschaftliche Untersuchungen, dass es die Bundesrepublik Deutschland gewesen sei, die in den 1960er-Jahren automatische Waffen im Sudan eingeführt habe. Im Jahre 1980 habe dann die damalige DDR darauf geantwortet, indem sie Kalaschnikows an die oppositionelle Sudanesische Volksbefreiungsarmee (Sudan People's Liberation Army, SPLA) geliefert habe. Somit hätten beide deutschen Staaten ihre Systemkonkurrenz nach Afrika getragen und dort eine verhängnisvolle Konfliktdynamik ausgelöst. ${ }^{53}$

Schaut man sich die politisch-militärische Entwicklung des Sudans in den ersten Jahrzehnten seiner Unabhängigkeit (1956) an, so ergibt sich ein deutlich anderes Bild. Bis 1967 dominierten Großbritannien und Frankreich beim Aufbau und bei der Ausrüstung der dortigen Landund Luftstreitkräfte, woran sich die Bundesregierung beteiligte, indem sie die Bewaffnung der damals zahlenmäßig noch begrenzten Streitkräfte mit G3-Gewehren und Maschinengewehren sicherstellte. Die Marine wurde mit Hilfe Jugoslawiens aufgestellt. Nach dem Sechs-Tage-Krieg im Juni 1967 brach die Regierung des Sudans die Beziehungen zu den westlichen Staaten aber ab und wandte sich der Sowjetunion zu. Erst diese rüstete die Streitkräfte massiv auf (unter anderem mit Panzern, Schützenpanzern und Kampfhubschraubern, schwerer und mittlerer Artil-

53 Vgl. Charlton Doki und Adam Mohamed Ahmad: Africa's arms dump. Following the trail of bullets in the Sudans, The Guardian, 2. 10. 2014. 
lerie sowie mit vielen kleinen und leichten Waffen). Über 600 Militärberater kamen damals aus der Sowjetunion und anderen sozialistischen „Bruderstaaten“ in das Land und bewirkten dadurch einen qualitativen Sprung, was die Fähigkeiten der sudanesischen Streitkräfte betraf. Die bis dahin knapp 18.000 Mann starken Streitkräfte wuchsen bis 1970 auf über 50.000 Mann an. Das bedeutete auch, dass das Sturmgewehr G3 nicht mehr das Standardgewehr der Streitkräfte bleiben konnte. Ihre erweiterten militärischen Fähigkeiten setzte die Regierung bei der Bekämpfung der Rebellenbewegung im Süden des Landes ein. Nach 1970 brach diese Allianz wieder auseinander und der Sudan erhielt in den Folgejahren Waffen, Ausrüstung und Ausbildungshilfe von den USA, die ihre Aktivitäten damit rechtfertigten, dass die Sowjetunion und deren Verbündeten das benachbarte Äthiopien und Somalia sowie die separatistischen Rebellen mit Waffen gegen den Sudan ausrüsteten. ${ }^{54}$ In den 1980er-Jahren nahm der Sudan dann eine zunehmend islamistische Ausrichtung und es wurde die Scharia eingeführt, was im nicht-muslimischen Süden des Landes auf Widerstand traf. In dieser Zeit kamen der Iran und China als primäre Waffenlieferanten ins Spiel.

Der Bürgerkrieg zwischen der Zentralmacht in Khartum und den Rebellen im Süden brach nicht deshalb aus, weil deutsche Gewehre vom Typ G3 diesen ansonsten friedlich verlaufenden Konflikt zum Eskalieren gebracht hätten, sondern weil die Zentralregierung eine mit Gewaltmitteln unterstützte Politik verfolgte, die die Peripherien des Landes marginalisierte und von diesen als unterdrückend empfunden wurde. Deutsche Waffen (vor allem G3-Gewehre) waren daran beteiligt, aber nicht in zentraler Rolle. Die Rebellen beschafften sich ihre Waffen weitgehend auf dem schwarzen Markt sowie durch Siege gegen sudanesische Regierungstruppen. ${ }^{55}$ Zudem halfen Äthiopien, Uganda und später auch Eritrea mit Waffen aus. Das bedeutete zwar, dass aufseiten der Regierung G3-Gewehre eingesetzt wurden, aber diese waren nur ein Teil eines sehr viel umfangreicheren und komplexeren Arsenals. Seit den 1980er-Jahren traten vornehmlich China und der Iran als Waffenlieferanten auf. Das G3-Gewehr wurde als Sturmgewehr spätestens in dieser Zeit von einem chinesischen Produkt abgelöst.

Der Konflikt endete 2005 mit einem Waffenstillstand und einem Friedensabkommen, an dessen Ende die Loslösung des Südsudans stand. Der Krieg dürfte zwischen 1983 und 2005 bis zu zwei Millionen Menschen das Leben gekostet haben, entweder direkt durch Waffeneinwirkung oder

54 Vgl. Eprile 1972, Wai 1979, Born 1980, Krause 1985a, Nna/Pabon/ Nkoro 2014.

55 Vgl. Nna/Pabon/Nkoro 2014. indirekt durch die Folgen des Krieges (Vertreibung, Krankheit, Hunger). Etwa 300.000 Tote soll es alleine durch die Einwirkung von Kleinwaffen gegeben haben. ${ }^{56}$ Seit 2013 ist im neu entstandenen Südsudan ein weiterer Bürgerkrieg ausgebrochen, bei dem bislang etwa 300.000 Menschen getötet und 3,5 Millionen vertrieben worden seien.

Schaut man sich Ergebnisse der Feldforschung des Small Arms Survey sowie der Conflict Armaments Research an, so bestätigt sich das Bild einer abnehmenden Bedeutung der G3-Gewehre und der deutschen Maschinengewehre in dem Konflikt. Sowohl die Untersuchung eroberter Waffen der sudanesischen Regierungstruppen wie die breitere Analyse der in den Konflikten verwendeten Arsenale lassen nicht erkennen, dass aus Deutschland stammende Waffen eine zentrale Rolle spielen. ${ }^{57}$ Die wesentlichen Ergebnisse der diesbezüglichen Analysen lassen sich wie folgt zusammenfassen:

- Bei den kleinkalibrigen Waffen ist eine deutliche Dominanz chinesischer Waffen $\mathrm{zu}$ erkennen und zwar durch das gesamte Spektrum, einschließlich der Sturmgewehre. ${ }^{58}$

- Von großer Bedeutung sind ebenfalls Kleinwaffen und Infanteriewaffen sowie Munition iranischer oder russischer Herstellung, wobei die Rolle Irans seit einigen Jahren abgenommen haben soll. ${ }^{59}$

- Sowohl bei der Munitionsherstellung für kleinkalibrige Waffen wie bei der Herstellung von Mörsern und deren Munition hat der Sudan ein hohes Maß an Selbstversorgung erreicht und exportiert diese auch in andere Länder. Darunter befindet sich auch Munition des Kalibers 7,62 × $51 \mathrm{~mm}$, das ist die Munition, die für das G3-Gewehr gebraucht wird. ${ }^{60}$ Zumindest dies ist ein Indikator dafür, dass G3-Gewehre im Land noch vorhanden sind und auch eingesetzt werden.

Dieser Eindruck verstärkt sich noch mehr, wenn man die Expertenberichte der Vereinten Nationen zur Überwachung der Waffenembargos gegen den Sudan und den Südsudan liest. Hier ist an keiner Stelle zu erkennen, dass Waffen deutscher Provenienz oder des Kalibers der G3-Gewehre noch eine Rolle spielen. Die wirkmächtigsten Einsatzwaffen sind hier Kampfhubschrauber und Kampfflugzeuge aus dem Bereich der früheren Sowjetunion (Russland, Ukraine) sowie Mehrfachraketenwerfer,

56 Vgl. Nna/Pabon/Nkoro 2014, 31.

57 Vgl. Leff/Le Brune 2014; Conflict Armaments Research 2017a und Conflict Armaments Research 2017b.

58 Vgl. Leff/Le Brune 2014, $43 \mathrm{ff}$.

59 Conflict Armaments Research 2017b.

60 Leff/Le Brune 2014, $60 \mathrm{ff}$. 
Artillerie, Haubitzen und Mörser. Kleine Waffen (Sturmgewehre, Gewehre, Maschinenpistolen, Pistolen) kommen weitgehend aus China oder aus Ländern des früheren Ostblocks. Aber auch der Iran sendet Waffen, Munition von einschlägigen NATO-Kalibern taucht nur noch selten auf. ${ }^{61}$

\subsection{Fallbeispiel Libyen}

Auch Libyen wird immer wieder als ein Beispiel genannt, wo deutsche Waffen in angeblich großen Mengen vorhanden sind und damit zu der Destabilisierung des Landes beigetragen hätten. Tatsächlich wurde nach dem Sturz des libyschen Diktators Muammer al-Qaddhafi im Sommer 2011 unter dem reichhaltigen Sortiment an leichten und schweren Waffen, welches dieser über Jahrzehnte angesammelt hatte, eine Kiste mit Sturmgewehren des Typs G36 der Firma Heckler \& Koch gefunden, die dort nicht hätten sein dürfen. Diese Kiste sorgte für Furore in den deutschen Medien und unter den Rüstungskritikern. Dass es ausschließlich die enormen Mengen an Waffen sowjetischen/ russischen, chinesischen, französischen, belgischen und italienischen Ursprungs sind, die heute tatsächlich zur Destabilisierung Libyens und des gesamten nordafrikanischen Raums beitragen, hat in der deutschen rüstungskritischen Debatte kaum Widerhall gefunden. Erkenntnisse von Small Arms Survey, von Conflict Armaments Research und anderen belegen die destabilisierende Rolle dieser Waffensysteme, insbesondere der Kleinwaffen, aber auch der tragbaren Luftabwehrsysteme. ${ }^{62}$ Deutsche Waffensysteme sucht man unter den dort belegten Funden jedoch vergebens. ${ }^{63}$

\subsection{Fallbeispiel Syrien-Krieg}

Im syrischen Bürgerkrieg kommen Waffen deutscher oder deutsch-französischer Gemeinschaftsproduktion vor. Aber sie nehmen ebenfalls eine marginale Rolle ein. Weder die syrischen Streitkräfte noch diejenigen der Freien Syrischen Armee oder der islamistischen Milizen kämpfen in größerem Stil mit Waffen, die aus Deutschland stammen. Die Bewaffnung des Islamischen Staates besteht größtenteils aus erbeuteten Kleinwaffen und Großwaffensystemen, die

61 Vgl. UN PoE Sudan 2013, 18-22; UN PoE Sudan 2014, 17-23; UN PoE Sudan 2015, 19-34; UN PoE Sudan 2016, 14-17; UN PoE 2017, S. 3-4; UN PoE South Sudan 2016, 25-33.

62 Spleeters 2013, Strazzari/Tholens 2014, Tessieres 2016 , Tessières 2018.

63 Conflict Armaments Research 2013, Conflict Armaments Research 2016c, Jenzen-Jones 2013, Jenzen-Jones/Rice 2016. zuvor der irakischen Armee gehörten und entweder russischen oder US-amerikanischen Ursprungs sind. Beachtlich ist auch der Sektor der selbst hergestellten Kleinwaffen und Sprengwaffen durch den Islamischen Staat. ${ }^{64}$

Mit dem Einschreiten der türkischen Streitkräfte in den Kurdengebieten Nordsyriens Anfang Januar 2018 hat sich insofern eine neue Lage ergeben, als dass hier Leopard 2A Panzer zum Einsatz kamen, die zuvor aus Deutschland an die Türkei exportiert worden waren. Was die Panzer allerdings in dem bewaldeten und schwer zugänglichen Berggebiet militärischen bewirken sollten, blieb unklar. Alle wesentlichen Kampfhandlungen fanden durch leicht bewaffnete Milizen statt, die Präsenz der Leopard 2A Panzer hatte bestenfalls symbolischen Wert (vielleicht war es auch eine Provokation der deutschen Bundesregierung).

\subsection{Die Bedeutung deutscher Kleinwaffen für den Drogenkrieg in Mexiko.}

Die mexikanische Regierung führt seit vielen Jahren eine Art „Krieg“ gegen das organisierte Verbrechen, dessen Akteure sich Dank der großen Einkünfte aus dem Drogenhandel massiv mit Waffen eingedeckt haben und über Milizen verfügen, die sich wie Guerilla- oder Terroristenorganisationen aufführen. Dieser Drogenkrieg hat in den Jahren 2015 und 2016 über 30.000 Todesopfer gefordert und gilt heute als einer der blutigsten innerstaatlichen Konflikte weltweit. ${ }^{65}$ Aufseiten der mexikanischen Polizei und des Militärs werden dabei auch Waffen deutscher Provenienz benutzt, die teilweise legal nach Mexiko gelangten, teilweise auf Wegen, die schwer nachzuvollziehen sind. Folgt man den Einschätzungen deutscher Rüstungskritiker, so sind diese Waffen die Hauptkampfmittel der Polizei und würden vermehrt auch von den kriminellen Milizen genutzt, nachdem sie diese erbeutet hätten. Damit würden deutsche Rüstungsexporte zur Eskalation des Konfliktes beitragen. ${ }^{6}$ Auch hier sieht die Realität anders aus. Zwei Dinge sind festzustellen: Zum einen sind die deutschen Waffen nur ein kleiner Teil der Ausrüstung der mexikanischen Polizei und des Militärs (der überwiegende Teil kommt aus den USA, hat seinen Ursprung aber in früheren Ländern des Ostblocks, wie Rumänien), zum

64 Vgl. Conflict Armaments Research 2014a, 2014b, 2015, und 2016a; s. a. C. J. Chivers: ISIS Ammunition shown to have Origins in USA and China, New York Times, 5. 10. 2014.

65 IISS 2017b, 5, und $344 \mathrm{ff}$.

66 Vgl. u. a. Hauke Friedrichs: Deutsche Waffen töten überall. ZEIT Online, 3. 2. 2014, http://www.zeit.de/politik/2014-02/illegale-waffen-heckler-und-koch; Steinmetz 2016. 
anderen lässt eine Auswertung der Herkunft von 100.000 durch die mexikanischen Sicherheitskräfte erbeuteten Waffen der kriminellen Milizen erkennen, dass Waffen deutscher Herkunft gerade mal 1,5 Prozent ausmachten. ${ }^{67}$

\subsection{Wirkungen deutscher Großwaffensysteme}

Ansonsten lassen sich über deutsche Kriegsschiffe und gepanzerte Fahrzeuge keine Hinweise finden, wonach diese zu Rüstungswettläufen oder zur Eskalation von zwischenstaatlichen Kriegen und Bürgerkriegen beigetragen hätten. Das ist bei den gelieferten Schiffseinheiten auch kaum vorstellbar, handelt es sich doch zumeist um U-Boote beziehungsweise um kleine oder mittlere Überwasserschiffe, die an Länder mit nachweisbar defensiven Absichten gehen. Aber auch selbst hier halten sich hartnäckig Fehlinformationen. Ein Beispiel ist die Lieferung der in Deutschland hergestellten U-Boote der Dolphin-Klasse an Israel, die seit den 1990er-Jahren der Mythos umgibt, sie seien Träger für israelische Kernwaffen und würden Israel eine nuklearstrategische Zweitschlagskapazität verleihen. ${ }^{68}$ Die U-Boote sind aber für derartige Waffensysteme technisch nicht ausgelegt und würden selbst bei Vornahme entsprechender technischer Veränderungen kaum dazu in der Lage sein, eine eigenständige nuklearstrategische Fähigkeit herzustellen. Der angebliche Marschflugkörper Turbo Popeye mit einer Reichweite von mehr als $1.500 \mathrm{~km}$ ist bislang nie getestet und nirgendwo gesichtet worden und selbst wenn es ihn gäbe, müsste er ein Gewicht haben, welches einen Ausstoßmechanismus erforderlich macht, der nicht in U-Boote dieser Größe passen würde. ${ }^{69}$

Es ist ohnehin festzustellen, dass die großen Kriege zwischen Staatenkoalitionen, bei denen über Jahre

67 Vgl. Boggs/Rand 2015; GAO 2016, 9.

68 Vgl. Grässlin 2013, 358 ff; bezeichnenderweise bezieht sich Grässlin als Hauptquelle auf die Deutsche Militärzeitschrift (DMZ), die dafür bekannt ist, dass sie einen rechtsradikalen Hintergrund hat. Des Weiteren glaubt er den Beweis dadurch erbringen zu können, dass er Journalisten des Nachrichtenmagazins Der SPIEGEL zitiert, die an Bord eines Dolphin-Bootes waren und seither behaupten, sie wüssten, dass dort Atomwaffen transportiert werden. Allerdings haben sie die angeblichen Atomwaffen nicht gesehen und ihre „Recherchen" bestanden weitgehend aus Interviews mit ehemaligen Amtsträgern, die mit dem Vorhaben nicht unmittelbar befasst waren, sondern nur ihre Vermutungen preisgaben. Der besagte SPIEGELArtikel bringt keinen einzigen Beleg für die These einer Atomwaffenfähigkeit, sondern ergeht sich nur in düsteren Spekulationen und der Wiedergabe von Zitaten; vgl. „Made in Germany“, SPIEGEL Nr. 23 vom 4. 6. 2012, Titelgeschichte, S. 20-33.

69 Näheres siehe Krause 2015. unter Einsatz aller vorhandenen Ressourcen gekämpft wird, mehr und mehr der Vergangenheit angehören. ${ }^{70}$ Von daher lassen sich keine Beispiele finden, bei denen Exporte deutscher Großwaffensysteme zu einer Eskalation beigetragen haben. Dies liegt auch an einer seit Jahrzehnten praktizierten Genehmigungspolitik aller Bundesregierungen, wonach sorgfältig darauf geachtet wird, dass die Regeln des Kriegswaffenkontrollgesetzes sowie des Außenwirtschaftsgesetzes eingehalten werden. Dazu gehören das Verbot der Unterstützung von Angriffskriegen ebenso wie die Orientierung an den außenpolitischen Interessen der Bundesrepublik Deutschland, die einen vorsichtigen Umgang mit Exporten von Waffen und Rüstungsgütern nahelegen, diese andererseits auch nicht kategorisch ausschließen (auch nicht in Spannungs- oder gar Kriegsgebiete). ${ }^{71}$

\section{Ausblick}

Geht man auf die eingangs gestellten Fragen zurück, so bleibt festzustellen, dass keine der in der politischen Debatte dominierenden Einschätzungen $\mathrm{zu}$ Größe, Umfang und Qualität deutscher Rüstungsexporte einer kritischen Prüfung standhält. Deutschland gehört zu den zehn größten Exporteuren von Kriegswaffen und sonstigen Rüstungsgütern, wobei es gegenwärtig den sechsten Platz einnehmen dürfte, nicht den dritten oder vierten. Was Exporte von kleinen Kriegswaffen betrifft, so ist der deutsche Anteil global gesehen minimal (unter einem Prozent) und man kann mit Sicherheit feststellen, dass Deutschland keine Rolle bei der Waffenversorgung der Konfliktparteien in innerstaatlichen Kriegen spielt.

Vergleicht man Deutschlands Position auf den Märkten für schwere, leichte und kleine Waffen mit der deutschen Position im weltweiten Handel, so fällt auf, wie groß der Unterschied ist. Im zivilen Export von Gütern liegt Deutschland nach China und den USA an dritter Stelle mit einem globalen Marktanteil von 8,2 Prozent. Bei Kleinwaffen und leichten Waffen dürfte der deutsche Anteil am weltweiten Handel im Promillebereich liegen, bei großen Waffensystemen und zentralen Waffenkomponenten bei Anteilen zwischen 2 und 4 Prozent.

Die in Deutschland geführte Debatte zu Rüstungsexporten ist traditionell mit großen Emotionen, phantastischen Spekulationen und exorbitanten Tartaren-

70 Vgl. Human Security Research Group 2014, Pettersson/Wallensteen 2015.

71 Vgl. Krause 2013. 
meldungen überladen. ${ }^{72}$ Das sollte in einer aufgeklärten Demokratie vermieden werden. In den 1970er- und 1980erJahren war vor allem die Geheimhaltungspraxis der Bundesregierung und vieler einschlägiger Firmen dafür verantwortlich, dass Spekulationen entstanden und abenteuerliche Verdächtigungen in die Welt gesetzt wurden. Heute ist die Lage anders: Die Bundesregierung hat sich längst zur Transparenz entschlossen und auch Firmen aus der wehrtechnischen Branche gehen sehr viel unverkrampfter mit der Thematik um. Die phantastischen Spekulationen und Verdächtigungen hören dennoch nicht auf. Heute sind es vor allem Rüstungskritiker und linkspopulistische Politiker und Politikerinnen, die die vorhandenen Daten dekonstruieren und dann zur Untermauerung abstruser Behauptungen und Verdächtigungen nutzen. Die vorhandenen Daten und Statistiken internationaler Forschungsinstitute werden bestenfalls selektiv zitiert. Es ist äußerst bedauerlich, dass in den deutschen Medien und auch unter den etablierten Parteien im Bundestag die nötige Distanz zur Dominanz dieser Einstellungsmuster fehlt. Der politische Diskurs in diesem Politikfeld ist von diesen Einstellungen in einem Maße vorstrukturiert, dass - um mit Immanuel Kant zu sprechen - der Ausstieg aus dieser „selbstverschuldeten Unmündigkeit“ eigentlich überfällig sein müsste. Zumindest verbale Abrüstung ist angesagt.

\section{Literatur}

Albrecht, Ulrich (1972): Politik und Waffengeschäfte. Rüstungsexporte in der BRD. München: Hanser.

Aken, Jan van / Lurz, Alexander (2012): Waffen für alle. Das Geschäft mit dem Tod, Blätter für deutsche und internationale Politik, 57 (3), 12-16.

Anders, Holger (2014): Identifying Sources. Small-calibre Ammunition in Côte d'Ivoire. Geneva: Small Arms Survey.

Arsovska, Anna (2014): Introduction: Illicit Firearms Market in Europe and Beyond, European Journal on Criminal Policy and Research, 20 (3), 295-305.

Auswärtiges Amt (2017): Jahresabrüstungsbericht 2016. Bericht der Bundesregierung zum Stand der Bemühungen um Rüstungskontrolle, Abrüstung und Nichtverbreitung sowie über die Entwicklung der Streitkräftepotenziale 2016. Berlin: Bundesregierung.

Berman, Eric G. (2012): Antitank Guided Weapons. Genf: Small Arms Survey.

Bevan, James (2013): Military Assault Rifles. Genf: Small Arms Survey.

Bevan, James (2014): Rifles. Genf: Small Arms Survey.

72 Zum Beispiel Grässlin 2013, Grässlin/Harrich/Harrich-Zandberg 2015; Friedrichs 2012.
Bitzinger, Richard A. (1994): The Globalization of the Arms Industry: The Next Proliferation Challenge, International Security, 19 (2), 170-198.

Bitzinger, Richard A. (2009): The European Defense Industry in the 21st Century. Challenges and responses, in: Bitzinger, Richard A. (Hrsg.): The Modern Defense Industry. Political, Economic and Technological Issues. Santa Barbara, Denver und Oxford; Praeger, 175-195.

Boggs, Clay/Rand, Kristen (2015): Gun-Running Nation. How Foreign-Made Assault Weapons are Trafficked from the United States to Mexico and What to Do About It. Washington, D.C.; Violence Policy Center/Washington Office on Latin America.

Born, Wolf-Ruthard (1980): Sudan - zehn Jahre Dschafer Mohamed Nimeiri. Europäische Wehrkunde, 29 (3), 137-147.

Bundesministerium für Wirtschaft und Energie (2016): Fakten zum deutschen Außenhandel. Berlin; Bundesregierung (Juni).

Bundesministerium für Wirtschaft und Energie (2017): Bericht der Bundesregierung über ihre Exportpolitik für konventionelle Rüstungsgüter im Jahre 2016. Berlin; Bundesregierung.

Conflict Armaments Research (2013): Rebel Forces in Northern Mali. Documented weapons, ammunition and related materiel April 2012-March 2013. London; CAR.

Conflict Armaments Research (2014a): Islamic State Weapons in Iraq and Syria. Analysis of weapons and ammunition captured from Islamic State forces in Iraq and Syria. London; CAR.

Conflict Armaments Research (2014b): Islamic State Ammunition in Iraq and Syria. Analysis of small-calibre ammunition recovered from Islamic State forces in Iraq and Syria. London; CAR.

Conflict Armaments Research (2015): Islamic State Weapons in Kobane. Analysis of weapons and ammunition captured from Islamic State forces in Kobane. London; CAR.

Conflict Armaments Research (2016a): Tracing the Supply of Components used in Islamic State IEDs. Evidence from a 20-month investigation in Iraq and Syria. London; CAR.

Conflict Armaments Research (2016b): Maritime Interdictions of Weapon Supplies to Somalia and Yemen. The Iranian Link. London; CAR.

Conflict Armaments Research (2016c): Investigating Cross-Border Weapon Transfers in the Sahel. London; CAR.

Conflict Armaments Research (2016d): Standardisation and Quality Control in Islamic State's Military Production. London; CAR.

Conflict Armaments Research (2017a): New Sudanese Weapons in Blue Nile State. London; CAR.

Conflict Armaments Research (2017b): Sudanese Stockpiles and Regional Weapons Diversion. London; CAR.

Connolly, Richard/Sendstad, Cecilie (2017): Russia's Role as an Arms Exporter. The Strategic and Economic Importance of Arms Exports for Russia. London; Chatham House.

Defense Science Board (1999): Final Report of the Defense Science Board Task Force on Globalisation and Security. Washington, D.C.; Department of Defense.

Demetriou, Spyros/Muggah, Robert/Biddle, Ian (2002): Small Arms Availability, Trade and Impacts in the Republic of Congo. Geneva: Small Arms Survey.

Devore, Marc R. (2013): Arms Production in the Global Village: Options for Adapting to Defense-Industrial Globalization, Security Studies, 22 (3), 532-572.

Dowdall, Paul (2004): Chains, networks and shifting paradigms. The UK defence industry supply system, Defence and Peace Economics, 15 (6), 535-550. 
Eprile, Cecil (1972): Sudan. The Long War. London: Institute for the Study of Conflict, Conflict Studies No. 12.

Farah, Douglas/Braun, Stephen Braun (2008): Merchant of Death: Money, Guns, Planes, and the Man Who Makes War Possible. New York; John Wiley \& Sons.

Florquin, Nicolas/Le Brun, Emilie/Leff, Jonah/Schroeder, Matt (2014): Documenting Weapons in Situations of Armed Conflict. Genf: Small Arms Survey.

Florquin, Nicolas/Seymour, Claudia (2016): Down, but not Out: The FDLR in the Democratic Republic of Congo. Genf: Small Arms Survey.

Friedrichs, Hauke (2012): Bombengeschäfte. Tod Made in Germany. München; Residenz Verlag.

Garcia, Denise (2009): Arms Transfers Beyond the State-to-State Realm, International Studies Perspectives, 10 (2), 151-68.

Garcia-Alonso, Maria D. C./Levine, Paul (2007): Arms Trade and Arms Races. A Strategic Analysis, in: Sandler, Todd/Hartley, Keith (Hrsg.): Handbook of Defence Economics, Vol. 2. Amsterdam; Elsevier, 941-971.

GKKE - Gemeinsame Konferenz Kirche und Entwicklung (2016): Rüstungsexportbericht der Gemeinsamen Konferenz Kirche und Entwicklung 2016. Bonn/Berlin: GKKE.

Grässlin, Jürgen (2013): Schwarzbuch Waffenhandel. Wie Deutschland am Krieg verdient. München: Heyne Verlag.

Grässlin, Jürgen / Harrich, Daniel / Harrich-Zandberg, Danuta (2015): Netzwerk des Todes. Die kriminelle Verflechtung von Waffenindustrie und Behörden. München; Heyne Verlag.

Guay, Terrence (1998): At Arm's Length. The European Union and Europe's Defence Industry. Houndsmill und London: MacMillan Publishers.

Guay, Terrence (2007): Globalization and its Implications for the Defense Industrial Base. Carlisle, PA; US Army War College Strategic Studies Institute.

Haftendorn, Helga (1971): Militärhilfe und Rüstungsexporte der BRD. Düsseldorf: Bertelsmann.

Haftendorn, Helga (1972): Militärhilfe im außenpolitischen Instrumentarium der BRD und der USA, Politische Vierteljahresschrift, 13 (3), 374-424.

Hanel, Dieter (2003): Die Bundeswehr und die deutsche Rüstungsindustrie. Bad Godesberg; Bernard \& Gräfe.

Hanel, Dieter (2012): Streitkräfte und Rüstung. Die Panzerindustrie. Bad Godesberg; Bernard \& Gräfe.

Hayward, Keith (2000): The Globalisation of Defence Industries, Survival, 42 (2), 115-132.

Hayward, Keith (2009): The Globalization of Defence Industries, in: Bitzinger, Richard A. (Hrsg.): The Modern Defense Industry. Political, Economic and Technological Issues. Santa Barbara, Denver und Oxford; Praeger, 107-122.

Hazen, Jennifer M./Horner, Jonas (2010): Small Arms, Armed Violence, and Insecurity in Nigeria. The Niger Delta in Perspective. Genf: Small Arms Survey.

Holton, Paul/ Pavesi, Irene (2017): Trade Update 2017. Out of the Shadows. Genf: Small Arms Survey.

Human Security Research Group (2014): Human Security Report 2013. The Decline in Global Violence. Evidence, Explanation, and Contestation. Vancouver: Human Security Press.

Inowlocki, Tania (2014): Feeding the Fire. Illicit Small Arms Ammunition in Afghanistan, Iraq and Somalia. Genf: Small Arms Survey.
International Institute for Strategic Studies - IISS (2017a): The Military Balance. The Annual Assessment of Global Military Capabilities and Defence Economics. London: Routledge.

International Institute for Strategic Studies - IISS (2017b): Armed Conflict Survey. The Worldwide Review of Political, Military and Humanitarian Trends in Current Conflicts. London: Routledge.

James, Andrew (2002): Comparing European responses to defense industry globalization, Defense and Security Analysis, 18 (2), 123-143.

Jenzen-Jones, N. R. (2013): Small Calibre Ammunition in Libya. Genf: Small Arms Survey.

Jenzen-Jones, N. R. (2014): Producers of Small Arms, Light Weapons and their Ammunition. Genf: Small Arms Survey.

Jenzen-Jones, N. R. (2015): Documenting Small Arms and Light Weapons. Genf: Small Arms Survey.

Jenzen-Jones, N. R. / Rice, Graeme (2016): The online Trade of Light Weapons in Libya. Genf: Small Arms Survey, Security Assessment in North Africa (SANA) Dispatch.

Jonsson, Michael / Brennan, Elliot (2014): Drugs, Guns and Rebellion: A Comparative Analysis of the Arms Procurement of Insurgent Groups in Colombia and Myanmar, European Journal on Criminal Policy and Research, 20 (3), 307-321

Karp, Aron (2103): Data Sources and the Estimation of Military-owned Small Arms. Geneva; Small Arms Survey.

Kasapoglu, Can / Ergun, Doruk (2016): From Low-Intensity Conflict To Hybrid Warfare: MANPADS at The Hands of PKK. Istanbul: EDAM Foreign Policy and Security Paper Series 2016/4.

Killicoat, Phillip (2007): Weaponomics. The Global Market for Assault Rifles. Washington, D.C.: The World Bank (Policy Research Working Paper 4202).

Krause, Joachim (1985a): Sowjetische Militärhilfe gegenüber Entwicklungsländern. Baden Baden; Nomos.

Krause, Joachim (1985b): Der internationale Handel mit konventionellen Waffen und Rüstungsgütern, Strukturen, Entwicklungen, Perspektiven. Ebenhausen; Stiftung Wissenschaft und Politik SWP-AZ 2439.

Krause, Joachim (2013): Gibt es eine Merkel Doktrin?, Internationale Politik, 68 (1), 100-105.

Krause, Joachim (2015): Fakt oder Phantasie? Lässt Israel in Kiel U-Boote für nuklear-strategische Aufgaben bauen?, Marine Forum, 90 (7-8), 13-15.

Lararevic, Jasna (2010): South-east European Surplus Arms. Genf; Small Arms Survey.

LeBrun, Emile / Rigual, Christelle (2016): Monitoring Arms Embargoes. Observations from Panels of Experts. Genf; Small Arms Survey.

Leff, Jonah / LeBrun, Emile (2014): Following the Thread. Arms and Ammunition Tracing in Sudan and South Sudan. Genf; Small Arms Survey.

Marsh, Nicholas (2002): Two Sides of the Same Coin? The Legal and Illegal Trade in Small Arms, Brown Journal of World Affairs, 9 (1), 217-228.

May, Channing (2017): Transnational Crime and the Developing World. Washington, D.C.; Global Financial Integrity.

Neal, Derrick J. / Taylor, Trevor (2001): Globalisation in the defence industry: An exploration of the paradigm for us and European defence firms and the implications for being global players, Defence and Peace Economics, 12 (4), 337-338. 
Nna, Nekabari Johnson / Pabon, Baribene Gbara/Nkoro, Friday (2012): Arms Proliferation and conflicts in Africa. The Sudanese Experiment, IOSR Journal of Humanities and Social Sciences, 4 (4), 31-39.

Paoli, Giacomo Persi / Aldridge, Judith/Ryan, Nathan / Warnes, Richard (2017): Behind the Curtain. The illicit trade of firearms, explosives and ammunition on the dark web. Santa Monica und Cambridge; RAND Corporation.

Parker, Sarah with Marcus Wilson (2016): A Guide to the UN Small Arms Process. 2016 Update. Genf; Small Arms Survey.

Pettersson, Therése / Wallensteen, Peter (2015); Armed Conflicts, 1946-2014, Journal of Peace Research, 52 (4), 536-550.

Pézard, Stephanie / Glatz, Anne-Kathrin (2010): Arms in and around Mauritania. National and Regional Security Implications. Genf; Small Arms Survey.

Rigual, Christelle (2013): Armed Groups' Holdings of Guided Light Weapons. Genf; Small Arms Survey.

Rigual, Christelle (2014): Armed Groups and Guided Weapons. 2014 Update with MENA Focus. Genf; Small Arms Survey.

Schroeder, Matt (2014): Fire and Forget. The Proliferation of Man-portable Air Defence Systems in Syria. Genf; Small Arms Survey.

Schroeder, Matt (2014): Rogue Rocketeers. Artillery Rockets and Armed Groups. Genf; Small Arms Survey.

Schroeder, Matt (2015): Missing Missiles. The Proliferation of Man-portable Air Defence Systems in North Africa. Genf; Small Arms Survey.

Schroeder, Matt / Lamb, Guy (2006): The Illicit Arms Trade in Africa: A Global Enterprise, African Analyst Quarterly, 1 (1), 69-78.

SIPRI - Stockholm International Peace Research Institute (2017): SIPRI Yearbook 2016. Armaments, Disarmament and International Security. Oxford; Oxford University Press.

SIPRI / FES / Berghof Stiftung (2013): SIPRI Yearbook 2013. Zusammenfassung auf Deutsch. Berlin: Berghof Stiftung.

SIPRI / FES / Berghof Stiftung (2016): SIPRI Yearbook 2016. Zusammenfassung auf Deutsch. Berlin: Berghof Stiftung.

Small Arms Survey (2007): Guns and the City. Yearbook. Genf; Small Arms Survey.

Small Arms Survey (2008): Risk and Resilience. Yearbook. Genf; Small Arms Survey.

Small Arms Survey (2009): Shadows of War. Yearbook. Genf; Small Arms Survey.

Small Arms Survey (2010): Gangs, Groups and Guns. Yearbook. Genf; Small Arms Survey.

Small Arms Survey (2011): States of Security. Yearbook. Genf; Small Arms Survey.

Small Arms Survey (2012): Moving Targets. Yearbook. Genf; Small Arms Survey.

Small Arms Survey (2013): Everyday Dangers. Yearbook. Genf; Small Arms Survey.

Small Arms Survey (2014): Women and Guns. Yearbook. Genf; Small Arms Survey.

Small Arms Survey (2015): Weapons of the World. Yearbook. Genf; Small Arms Survey.

Smith, Chris (2003): In the Shadow of a Cease-Fire. The Impact of Small Arms Availability and Misuse in Sri Lanka. Genf; Small Arms Survey.

Spleeters, Damien (2013): FAL-Rifles in Libya. Genf; Small Arms Survey.
Steinmetz, Christopher (2016): Kleinwaffen in Kinderhänden. Deutsche Rüstungsexporte und Kindersoldaten. Berlin; Berliner Informationszentrum für transatlantische Sicherheit (BITS).

Strazzari, Francesco / Tholens, Simone (2014): Tesco for Terrorists' Reconsidered: Arms and Conflict Dynamics in Libya and in the Sahara-Sahel Region, European Journal on Criminal Policy and Research, 20 (3), 343-360.

Tarr, Gregory L. (2015): MANPADS. Proliferation Reduction by Design. Genf; Small Arms Survey.

Tessières, Savannah (2016): Measuring Illicit Arms Flows - Niger. Genf; Small Arms Survey.

Tessières, Savannah (2018): At the Crossroads. Insecurity, Terrorism, and Arms Trafficking in Niger. Genf: Small Arms Survey.

Thachuk, Kimberley / Saunders, Karen Saunders (2014): Under the Radar: Airborne Arms Trafficking Operations in Africa, European Journal on Criminal Policy and Research, 20 (3), 361-378.

Theohary, Catherine A. (2015): Conventional Arms Transfers to Developing Nations, 2007-2014. Washington, D.C.; Congressional Research Service.

Theohary, Catherine A. (2016): Conventional Arms Transfers to Developing Nations, 2008-2015. Washington, D.C.; Congressional Research Service, December 19, 2016

Udis, Bernard (2009): Offsets and International Industrial Participation, in: Bitzinger, Richard A. (Hrsg.): The Modern Defense Industry. Political, Economic and Technological Issues. Santa Barbara, Denver und Oxford; Praeger, 257-271.

UN PoE South Sudan (2016): Final Report of the Panel of Experts on South Sudan established pursuant to Security Council resolution 2206 (2015), Un Doc. S/2016/70, 22. Januar.

UN PoE Sudan (2013): Report of the Panel of Experts on the Sudan established pursuant to resolution 1591 (2005), UN Doc. $\mathrm{S} / 2013 / 79$ vom 12 . Februar.

UN PoE Sudan (2014): Report of the Panel of Experts on the Sudan established pursuant to resolution 1591 (2005), UN Doc. S/2014/87 vom 11. Februar.

UN PoE Sudan (2015): Report of the Panel of Experts on the Sudan established pursuant to resolution 1591 (2005), UN Doc. S/2015/31 vom 19 Januar.

UN PoE Sudan (2016): Final Report of the Panel of Experts on the Sudan established pursuant to resolution 1591 (2005), UN Doc. S/2016/805 vom 22. September.

UN PoE Sudan (2017): Final Report of the Panel of Experts on the Sudan established pursuant to resolution 1591 (2005), UN Doc. S 2017/22 vom 9. Januar.

United States Government Accountability Office (2016): Firearms Trafficking. U.S. Efforts to Combat Firearms Trafficking to Mexico Have Improved, but Some Collaboration Challenges Remain. Washington, D.C.; GPO.

Wai, Dunstan M. (1979): The Sudan. Domestic Politics and Foreign Relations under Nimeiry, African Affairs - The Journal of the Royal African Society, 76 (31), 297-317

Walsh, Lawrence E. (1993): Final Report of the Independent Counsel for Iran/Contra Matters. Volume I. Investigations and Prosecutions. United States Court of Appeals for the District of Columbia Circuit, zu finden unter https://fas.org/irp/offdocs/ walsh/source.htm. 